\title{
Comparing virulence of Cryphonectria parasitica isolates recovered from portions of cultures or cankers established before versus those after hypovirus innoculations
}

William E. Jones

West Virginia University

Follow this and additional works at: https://researchrepository.wvu.edu/etd

\section{Recommended Citation}

Jones, William E., "Comparing virulence of Cryphonectria parasitica isolates recovered from portions of cultures or cankers established before versus those after hypovirus innoculations" (2008). Graduate Theses, Dissertations, and Problem Reports. 4387.

https://researchrepository.wvu.edu/etd/4387

This Thesis is protected by copyright and/or related rights. It has been brought to you by the The Research Repository @WVU with permission from the rights-holder(s). You are free to use this Thesis in any way that is permitted by the copyright and related rights legislation that applies to your use. For other uses you must obtain permission from the rights-holder(s) directly, unless additional rights are indicated by a Creative Commons license in the record and/ or on the work itself. This Thesis has been accepted for inclusion in WVU Graduate Theses, Dissertations, and Problem Reports collection by an authorized administrator of The Research Repository @ WVU. For more information, please contact researchrepository@mail.wvu.edu. 
Comparing Virulence of Cryphonectria parasitica Isolates Recovered from Portions of Cultures or Cankers Established Before Versus Those After Hypovirus Innoculations

\author{
William E. Jones
}

\begin{abstract}
Thesis submitted to the
College of Agriculture, Forestry and Consumer Sciences

at West Virginia University

in partial fulfillment of the requirements

for the degree of
\end{abstract}

\begin{abstract}
Master of Science
in

Plant \& Soil Sciences
\end{abstract}

William L. MacDonald, Ph.D., Chair

Alan R. Biggs, Ph.D.

Frederick V. Hebard, Ph.D.

James B. Kotcon, Ph.D.

Division of Plant and Soil Sciences

Morgantown, West Virginia

2008

Keywords: biocontrol, chestnut blight, hypovirus 


\title{
ABSTRACT \\ Comparing Virulence of Cryphonectria parasitica Isolates Recovered from Portions of Cultures or Cankers Established Before Versus Those After Hypovirus Innoculations
}

\author{
William E. Jones
}

\begin{abstract}
Variable recovery of hypovirulent (HV) and virulent (V) isolates from repeatedly sampled, identical chestnut blight cankers, caused by Cryphonectria parasitica, prompted this study to monitor more closely the isolate recovery over time to determine hypovirulent or virulent status. To meet the objective, laboratory and field experiments were conducted between 1998 and 2000 to recover isolates from cultures and cankers challenged with HV inoculum. Cultural studies demonstrated 1) hypovirus transfer readily occurred to actively growing colony margins, and 2) that established, virulent mycelium one-to-six weeks-old at time was unable to acquire hypovirus. Results from field studies demonstrated a significant difference in recovery of $\mathrm{HV}$ isolates obtained from older portions versus younger portions of cankers. The greatest recovery of HV isolates was from mycelium established just after challenge $(44.4 \%)$ versus $4.6 \%$ of samples HV from the portions of cankers established four months prior to challenge. When bark plugs were sub-divided into different tissues, $20 \%$ contained both virulent and hypovirulent mycelium. Based upon this work there appears to be at least one hypothesis to explain variable recovery of $\mathrm{HV}$ and $\mathrm{V}$ isolates from identical cankers sampled over time: that rather than conversion of existing mycelium to HV status, recovery of HV isolates indicates that the subsequent fungal growth happened to be hypovirulent.
\end{abstract}




\section{ACKNOWLEDGEMENTS}

I would like to thank the many people that were involved in this thesis and my graduate project. First, I would like to thank my advisor Dr. William MacDonald for his patient guidance and help throughout this research project. Large thanks are due for Mark Double who kept the laboratory running and always had the answers or at least a song to any of my questions. I also thank Dr. Desta Fekedulegn for performing the statistical analyses. Paul Chaloux is due many thanks for accompanying me for all of my fieldwork and as an always arguing but always listening, fellow graduate student. I also thank the other committee members, Drs. Alan Biggs, Fred Hebard, and James Kotcon for offering help, suggestions and advice whenever it was needed. Finally, thanks and love for Amy and Alexandra for the support and happiness they have given me over the years. 


\section{TABLE OF CONTENTS}

Abstract $\quad$ ii

Acknowledgements $\quad$ iii

Table of Contents $\quad$ iv

List of Figures $\quad$ V

List of Tables $\quad$ vii

Introduction 1

Literature Review 3

Chapter 1 - Acquisition of Hypovirus in vitro 9

Methods and Materials $\quad 9$

$\begin{array}{ll}\text { Results } & 20\end{array}$

Chapter 2 - Acquisition of Hypovirus in vivo 30

$\begin{array}{ll}\text { Methods and Materials } & 30\end{array}$

Results 33

Formula 1: Percent of samples orange-pigmented virulent 36

Chapter 3 -Acquisition of Hypovirus in vivo 42

Methods and Materials $\quad 42$

Results 46

Discussion $\quad 52$

$\begin{array}{ll}\text { Conclusions } & 65\end{array}$

$\begin{array}{ll}\text { Literature Cited } & 66\end{array}$

Appendix A: Method for Verification of Phenotypic Data from Culturing Work 69

Appendix B: Growth Media Formulations 72

$\begin{array}{ll}\text { Curriculum Vitae } & 73\end{array}$ 


\section{LIST OF FIGURES}

Figure 1: Isolates utilized throughout this study

Figure 2: Figure 2: Cartoon depicting hypovirus challenge arrangements. [Challenges were made as co-inoculations, at the leading edge of expanding colonies or opposite of the colony initiation point. The orange square represents the colony initiation point and the brown square represents the hypovirus challenge point.]

Figure 3: Example of the photocopy records maintained of plates after sampling. [Numbers written on the plates indicates the number of samples isolated from those colonies.] 16

Figure 4: Cartoon depicting hypovirus challenges as conducted in Experiment I-B. [Challenges consisted of co-inoculations, challenges to the colony leading edges, and challenges made behind colony leading edges.]

Figure 5: Example of the twelve isolates recovered from a one-week-old colony (upper left) challenged at the colony margin one week prior. [Note: samples 1-5 \& 8 are darkly pigmented (orange-pigmented virulent) and colonies do not fuse whereas samples 6, 7, 9-12 are lightlypigmented (orange-pigmented hypovirulent) and colonies fused.]

Figure 6: Percent orange-pigmented virulent (OV), orange-pigmented hypovirulent (OHV) and contaminated (C) isolates recovered from mycelium that had expanded from 1-to-6 weeks before (-) to 1-to-3 weeks after $(+)$ challenge.

Figure 7: Percent and linear trends of orange-pigmented virulent (OV) and hypovirulent (OHV) isolates recovered from co-inoculated colonies of YB-2 and 80-2C [CHV1 80-2] over six weeks of sampling.

Figure 8: Percentage and linear trends of orange-pigmented virulent (OV) and orangepigmented hypovirulent $(\mathrm{OHV})$ isolates recovered from one-week-old colonies challenged at the colony leading edge over six weeks of sampling.

Figure 9: Percentage and linear trends of orange-pigmented virulent and orange-pigmented hypovirulent isolates recovered from two-week-old colonies challenged at the colony leading edge over six weeks of sampling.

Figure 10: Summary of data from three weeks of sampling of the percent orange-pigmented virulent and orange-pigmented hypovirulent isolates recovered from colonies when hypovirulent inoculum challenges were made as co-inoculations ( 0 weeks old), challenge to colony margins (1 week old), and challenges behind colony margins (1 week old).

Figure 11: Bone marrow biopsy tool and microtiter dish utilized to obtain and transport bark plug samples to the laboratory, respectively. 
Figure 12: Recovery of orange-pigmented hypovirulent (OHV), orange-pigmented virulent (OV) or contaminant (C) isolates from cankers over all ten sample dates.

Figure 13: Percent of orange-pigmented hypovirulent (OHV) isolates from May 1999 thru February 2000 sample dates from portions of cankers established before versus after hypovirus challenge (number on tops of bars gives the number of samples each bar represents).

Figure 14: Percent of orange-pigmented hypovirulent (OHV) isolates per outlined canker ring recovered from cankers throughout the course of the study.

Figure 15: Frequency of orange-pigmented hypovirulent (OHV), orange-pigmented virulent (OV), and contaminated (C) isolates recovered from Experiment II-A versus Experiment III-A cankers.

Figure 16: Mean number and standard error of orange-pigmented hypovirulent (OHV) isolates recovered from inner bark, outer bark and cambium-xylem.

Figure 17: Mean number and standard error of orange-pigmented virulent (OV) isolates recovered from inner bark, outer bark and cambium-xylem host tissues.

Figure 18: Digital scan of the RT-PCR products of $1 \mathrm{~Kb}$ plus DNA ladder (Lanes 1 and 16), standard virulent and hypovirulent isolates (Lanes 2, 3 and 17) and phenotypically virulent and hypovirulent isolates (Lanes $4-15$ ) on $0.8 \%$ agarose gel.

Figure 19: Digital scan of the RT-PCR products of $1 \mathrm{~Kb}$ plus DNA ladder (Lanes 2 and 17), standard virulent and hypovirulent isolates (Lanes 1, 15 and 16) and phenotypically virulent and hypovirulent isolates (Lanes 3-14) on $0.8 \%$ agarose gel. 


\section{LIST OF TABLES}

Table 1: Cryphonectria parasitica isolates and hypoviruses utilized throughout this project.

Table 2: Table 2: The total number of orange-pigmented virulent (OV), orange-pigmented hypovirulent (OHV), brown-pigmented hypovirulent (BRHV), and contaminated (C) isolates recovered from mycelium that was established from 6 weeks before $(-)$ to 3 weeks after $(+)$ challenge.

Table 3: Diameter at breast height (DBH) and bark thickness and rating of trees used for this study (*Cankers on these trees were not treated with a hypovirulent strain and served as controls).

Table 4: Average canker length and width (cm) of cankers treated with hypovirulent (HV) versus virulent (V) inoculum.

Table 5: Mean area $\left(\mathrm{cm}^{2}\right)$ of cankers treated with brown-pigmented hypovirulent (HV) versus brown-pigmented virulent (V) inoculum.

Table 6: Mean number of $\mathrm{OHV}, \mathrm{OV}$ and $\mathrm{C}$ isolates recovered per canker from Samples 1 to 6 (Nov-98 to Apr-99) versus Samples 7 to 10 (May-99 to Feb-00).

Table 7: Percent of samples scored orange-pigmented hypovirulent (OHV) from outlined monthly canker expansion rings from all sample dates.

Table 8: Recovery of orange-pigmented virulent (OHV), orange-pigmented virulent (OV), and contaminated $(\mathrm{C})$ isolates from outer bark, inner bark, and cambium-xylem tissues from 344 dissected bark plugs.

Table 9: Isolates recovered from unchallenged cankers (Experiment III-C) on the dates of April 26, 1999 and February 25, 2000. 


\section{INTRODUCTION}

The introduction of Cryphonectria (Endothia) parasitica (Murr.) Barr from Asia to North American in the late 1800's reduced the American chestnut (Castanea dentata (Marsh.) Borkh.) from its dominant canopy position to a state of perpetual re-sprouting. C. parasitica is the causal agent of chestnut blight, a disease typified by diffuse stem and branch cankers on susceptible trees. Chestnut blight now represents a classical example of the problem associated with an introduced species; without time for co-evolution between a host and pathogen little opportunity exists for development of disease resistance making disease inpacts severe.

The chestnut blight fungus grows in tree bark, cambium and even xylem tissues in the form of mycelial fans in newly killed host tissue or as individual hyphae in dead cells. Infection results in direct tissue death and also can lead to extensive mechanical blockages by stimulating over-production of tyloses within xylem vessels. Following canker formation, distal portions of the stems typically wilt, or "blight," followed by rapid death. To date, all attempts to control the disease and restore the American chestnut to its former status as a dominant forest canopy species have failed.

Introduction of $C$. parasitica to Europe led to a similar disease development on the European chestnut (Castanea sativa Mill.) as it had in North America. However, by 1951, disease progress shifted from the formation of lethal cankers to the development of non-lethal, superficial infections, enabling the trees to callous over the cankers. Studies of these non-lethal cankers yielded abnormal isolates of $C$. parasitica. In contrast to typical virulent isolates, isolations taken from callousing cankers yielded cultures of $C$. parasitica with reduced pathogenicity and altered levels of growth, pigmentation and sporulation. The reduction in the ability of the isolates to cause disease was later termed hypovirulence. Further work indicated hypovirulence was a feature of the cytoplasm that could be transferred and conferred to other cultures via hyphal anastomoses. The phenomenon of hypovirulence was later determined to be the result of fungal infections by biotrophic viral parasites, called hypoviruses. Because these hypoviruses are capable of debilitating Cryphonectria they are thought to be responsible for the recovery of European chestnut from chestnut blight. 
The level of disease control hypoviruses afforded to European chestnut generated interest for testing their usefulness as biological control agents on American chestnut. Despite numerous biological control attempts using hypoviruses, most trials located in the native range of American chestnut have met with limited success. The primary explanation as to why hypoviruses have been unsuccessful in North America is based on the system of vegetative compatibility within the fungus. This system acts to limit the spread of hypoviruses by reducing the potential for hyphal anastomoses between virulent and hypovirulent strains when the two strains are vegetatively incompatible. In Europe, reduced diversity of vegetative compatibility genes exists, which may explain why hypoviruses appear to spread more efficiently.

Little is known about the process by which hypoviruses colonize host mycelium and how they develop in cankers over time. Hypoviruses appear to be transmitted to younger portions of cankers first, colonizing the outer margin of the canker, and only later progressing toward the canker center. Repeated sampling of the same areas of cankers has shown a variable pattern in the recovery of virulent and hypovirulent isolates.

The lack of understanding about how hypoviruses are transmitted and colonize cankers prompted this research. The overall objective was to evaluate the influence of mycelial age on the transmission and colonization of hypoviruses within mycelium and cankers. Three different approaches were used to meet the objective. The first approach was a laboratory experiment to determine the age at which mycelium was no longer able to acquire hypovirus. The second was a field experiment involving repeatedly sampling a set of cankers over the course of one year to determine if mycelium in younger portions of cankers becomes hypovirus-infected prior to mycelium in older portions. A final field study involved dissecting cankers to determine if mycelium in different layers of phloem becomes equally infected with hypoviruses. 


\section{LITERATURE REVIEW}

American chestnut (Castanea dentata) was reduced from a dominant forest canopy tree to an understory shrub by an exotic fungus (Anagnostakis, 1987) in what was one of the most ecologically significant natural occurrences to adversely affect the hardwood forests of eastern North America since the last ice age. Cryphonectria parasitica is the causal agent of the chestnut blight disease. The parasite grows in the bark, xylem and cambial regions forming pale brownish mycelial fans leading to ellipsoid-shaped perennial, lethal cankers (Anderson and Rankin, 1914). Infections to a depth of four-to-five annual growth rings in the xylem have been demonstrated (Anderson, 1913). Cankers lead directly to the death of infected trees from cambial death and clogging of the xylem from excessive tyloses blockages (McManus, 1989). However, the actual mechanisms leading to xylem dysfunction are unclear. The fungus is especially fast growing in the bark of susceptible trees. The browned and wilted, or "blighted" leaves give the disease its name. All species in the genus Castanea [e.g. Japanese chestnut (C. crenata Seib. and Zucc.), Chinese chestnut (C. mollissima B1.), chinquapin (C. pumila (L.) Mill), and European chestnut (C. sativa)] may serve as hosts for the pathogen, but there are varying levels of resistance in some varieties. $C$. parasitica also has been shown to cause cankers and live saprophytically on a number of Quercus species (Stipes et al., 1978).

This introduced pathogen was first discovered in the United States in New York City in 1904 at the New York Zoological Park. The discovery was made by garden staff member H. W. Merkel who noticed something was killing the chestnut trees. That $C$. parasitica was the pathogenic agent causing the problem was easily demonstrated by

fulfilling Koch's Postulates (Murrill, 1906). However, it appears that rather than a single source of infection from the New York Zoological Park, the fungus likely had been established for a number of years prior to 1904 in planted stock of Oriental descent (Anagnostakis, 1993). Haven Metcalf implicated Bedford County, Virginia and Baltimore County, Maryland as original centers of infection due to the extent of disease incidence, their large number of planted oriental chestnut trees, and the age of those infections (Anderson and Rankin, 1914). Regardless of the number of original infection 
centers, within 40 years of its first discovery, almost every large chestnut tree in the natural range of the species was dead or dying.

Prior to the chestnut blight epidemic there were approximately 3.5 billion standing chestnut stems (Roane et al., 1986). This would have amounted to 200,000,000 acres of pure chestnut trees (Kuhlman, 1978). The economic losses to the disease at the time of the epidemic were staggering but losses in economic forest productivity continue to this day due to replacement of chestnut with less economically desirable species such as red maple (Abrams, 1989).

Despite near total destruction of the standing volume of chestnut trees, the blight did not lead to extinction because the fungus seems relatively incapable of successfully invading root tissues. Most likely, the soil microflora is antagonistic to the fungus thus preserving the root system from the ravages of the blight fungus (Weidlich, 1978). The ability of chestnut to grow a profusion of sprouts after the stem has been killed has ensured continued survival of the species at some sites despite survival of $C$. parasitica in the forest ecosystem (MacDonald and Fulbright, 1991).

The early work on growth of chestnut cankers and pathogen life cycle was completed shortly after report of the disease's outbreak in New York (Anderson, 1914). Cryphonectria was determined to be a wound parasite requiring fresh wounds that kill living cells, as shallow wounds in outer bark and certain insect holes inoculated with the pathogen were not shown to yield infections (Rankin, 1914). Initial invasions of the bark began with a massing of hyphae in a wound into a flattened mycelium. Any type of wound that extends deeper than the outer green cortex of the bark has been shown to be sufficient for infections (Anderson and Babcock, 1913).

Mycelium penetrates into surrounding bark tissues in characteristic "mycelial fans", and not as single hyphae in primary advancement into living tissues (Bramble, 1936). Secondary invasion of bark takes place as single hyphae that penetrate parenchyma cells behind the leading edge of the mycelial fans. Apparently, advancement of mycelial fans is the result of mechanical pressure, and not enzymatic degradation of cells as it advances. The fans varied in length from a millimeter to three centimeters in length. The individual hyphae in the fans were seen to branch only sparsely and to be comparatively more uniform in diameter than when they are grown in "agar culture" 
(Anderson, 1913, and Anderson and Rankin, 1914). Further, hyphae are contained in individual "ray branches" that do not anastomose.

Cankers are the result of the trees responding to the presence of the pathogen. The rate and extent of mycelial fan development appears to be the most important factor that determines the extent of canker growth and enlargement (Hebard et al., 1984). Mycelial fans are produced only during the growing season. Growth on dormant stems has been referred to as saprophytic (Rankin, 1914). However, canker enlargement during winter has been shown, but no "fresh-looking" new fans were produced.

While different species and varieties of chestnut trees may differ in their susceptibility to the disease, it appears that susceptibility derives from the ability of the fungus to obtain nutrients without resistance from host cells (Hebard et al., 1984). Other factors such as wound periderm that restrict further fungal advancement to the cambium result in "superficial cankers," which were an expression of resistance as such infections do not lead to lethal bark girdling. Early studies showed hypertrophy of cankers only when wound periderm had successfully isolated the entire infected area (Bramble, 1936).

By examining changes in each major tissue and cell type in the primary cortex, the pericycle, the bast zone, the cambium, and outer xylem (no fungal invasion noted) from fungal actions, it was shown that the fungus does not utilize or invade the bark tissues equally (Keefer, 1914). In the primary cortex, cork cells are only changed by mechanical pressure by fruiting bodies, collenchyma cells also are crowded by fruiting bodies but become lignified, parenchyma cells harbor mycelium inside their cell walls, and sclerenchyma cells are totally unaffected. Other tissue types yielded similar results; food storing cells as well as dividing and growing cells tended to be destroyed, while scleritized or lignified strengthening cells are relatively less susceptible (Keefer, 1914). Xylem infections were found to penetrate to a maximum of $12 \mathrm{~mm}$ and five annual growth rings of stem tissue of a stem-girdling canker.

By 1938 the blight was found to have spread to Europe (Biraghi, 1946). The disease epidemic spread as rapidly as it had in North America, and similar levels of destruction experienced in North America were expected for the European chestnut (Castanea sativa). However, a unique natural biological control phenomenon termed "hypovirulence" was found to be actively protecting the European chestnut trees from the 
blight fungus (Heiniger and Rigling, 1994). Coupled with the somewhat more resistant host trees, chestnut blight in Europe was not nearly as detrimental as it was in North America (Turchetti, 1978).

Hypovirulence, as loosely defined means "any state of disease-producing capacity" that is less than the norm for that disease, or reduced virulence (Elliston, 1982). Using methionine and lysine auxotrophic strains as markers to determine transmission it was discovered that the phenomenon of hypovirulence is caused by "cytoplasmic determinants" transferred by hyphal anastomosis (cytoplasmic union of two or more hyphae) from hypovirulent to virulent strains in both culture and host tissue (Van Alfen et al., 1975). Hypovirulence was associated with infection with double-stranded RNA (dsRNA) with a range in genome size and that the dsRNA was extractable (Day, 1977). Difficulties in working with dsRNA were noted ranging from incomplete transmission to conidia (Day, 1977) to defective genome elements arising from various internal deletions (Hillman et al., 2000 and Shapira et al., 1991).

Infection of filamentous fungi by dsRNA viruses have been described from a wide range of species including Aspergillus (Varga et al., 1998) and Rhizopus (Papp et al., 2001). Interestingly, dsRNA hypoviruses appear more closely related to ssRNA plant potyviruses than dsRNA viruses (Koonin et al., 1991). Unlike typical viruses, hypoviruses are not discretely packaged or capable of infecting new hosts extracellularly; rather they are contained in vesicles and rely on cytoplasmic fusion of hyphae for transfer (Hansen et al., 1985).

Hypovirulent and virulent isolates of the fungus often create cankers with different morphologies, although the presence of dsRNA has not been definitively linked to canker morphology (McManus, 1989). Virulent cankers typically have been characterized as having necrotic bark that is sunken in appearance and bearing abundant orange fructifications. Conversely, hypovirulent cankers often appear irregularly swollen and lack abundant fruiting bodies. Although $C$. parasitica has been shown to colonize xylem, only virulent isolates have been shown to be capable of xylem colonization (McManus, 1989). The xylem underlying virulent cankers was reported to be nonfunctional and of differing anatomy from xylem underlying hypovirulent cankers. McManus (1989) also suggested that the American chestnut trees recovering in Michigan 
may owe much of their health to the functioning xylem underlying the hypovirulent cankers.

The distribution of hypoviruses in cultures and cankers is poorly understood. One impediment to making generalizations about localization of hypovirulent mycelium within cankers arises from differences in methodologies among studies. Some experiments were based on methodologies in which sample locations were assigned randomly (Robbins and Griffin, 1999) while others were based on a more stratified sampling arrangement at canker margins (Balbalian, 1998 and Shain and Miller, 1992). Also, differences in the methods to introduce hypovirulent inoculum to cankers exist as well as differences in time of season. Previous findings indicate that sampling cankers in the same general spot and arrangement over time yields varying levels of hypovirus recovery (Balbalian, 1998) and that the same bark sample can yield multiple isolates that differ in virulent-hypovirulent phenotype (Griffin, 1999). Canker margins were shown to be colonized within three week's after hypovirulent inoculations with delayed conversion of interior portions of cankers (Shain and Miller, 1992). However, even one year after hypovirulent-inoculum challenge, cirrhi repeatedly sampled to obtain conidia for culture continued to yield virulent inoculum even when the underlying mycelium yielded hypovirulent isolates (Shain and Miller, 1992). This was still referred to as [mycelial] “conversion". In one detailed study where sample locations were arranged in a 7 x 7 lattice the recovery of hypovirulent and virulent isolates was found to be random based on frequency and spatial statistics and lattice cells could contain both pigmented (virulent) and white phenotype (hypovirulent) isolates (Griffin, 1999).

Hypoviruses in the genus Hypovirus Cryphonectria hypovirus are contained within their host hyphae in approximately 50-90 nm diameter pleomorphic lipid vesicles (Dodds, 1980) as naked dsRNA (Hansen, et al., 1985). The vesicles are thought to be of host-origin as they are composed of the same sugars as fungal cell walls and similar vesicles are found in both virulent and hypovirulent strains of $C$. parasitica (Hansen, et $a l .$, 1985). Ultrastructural examinations of fungal hyphae revealed that vesicles are membrane-bound and are associated with Golgi cisternae indicating they were formed in place and not translocated there (Newhouse and MacDonald, 1983). 
Similarly to rapid canker margin colonization by hypovirus, in situ hybridization techniques using cDNA probes previously have been used to quantify movement of hypoviruses between cultures. Using these probes, hypoviruses were shown to rapidly colonize and were predominately concentrated in the growing colony margins (Martin and Van Alfen, 1991). Colonization of the perimeter of the colonies (30 mm/day) was presumed to be the result of lateral transmission through connected hyphae rather than hyphal growth (4.4 mm/day average) (Martin and Van Alfen, 1991). Rapid lateral hypovirus transmission is confusing since passive translocation of hypovirus through anastomoses has been discounted because of the association of Golgi cisternae with virus-like particles (Newhouse and MacDonald, 1991).

The severity of chestnut blight coupled with the value people had placed on chestnut prior to the disease has resulted in sustained efforts at attempts to restore chestnut to the North American forests. Attempts at biocontrol have met with limited success, possibly due in part to structural barriers such as vegetative incompatibility but perhaps also due to limited understanding of fungal dynamics within cankers over time. Therefore, the goal of this project was to add to the understanding of the chestnut blight pathosystem by characterizing isolate recovery over time from Cryphonectria parasitica cultures and cankers. To meet the goal, several experiments were conducted between 1998 and 2000 for the purpose of recovering fungal isolates for culture. 


\section{CHAPTER I-ACQUISITION OF HYPOVIRUS IN VITRO METHODS AND MATERIALS}

Two laboratory experiments were conducted to evaluate the effect of mycelial age on transmission and colonization of hypoviruses over time. In the first experiment (Experiment A), cultures ranging in age from 0 to 6 weeks were challenged with an isolate infected with a European hypovirus, then sampled for 6 weeks to monitor hypovirus colonization. The second experiment (Experiment B) was conducted in a similar manner, but an additional challenge method (challenge behind colony leading edges) was made and the experiment duration was shorter. In Experiment B challenges were made with a strain infected with a North American hypovirus, a strain infected with a European hypovirus, or a virulent control.

The experimental work initially was to be completed using $150-\mathrm{mm}$ diameter petri dishes because larger dishes were thought to allow for a longer growth period for testing mycelial interactions. However, repeated attempts to maintain cultures over long time periods were unsuccessful due to excessive contamination. Therefore, all laboratory work was completed using 100-mm diameter petri dishes.

\section{A. Cryphonectria parasitica isolates}

Isolates infected with a European-type and a North American-type hypovirus were used in this study (Table 1, Figure 1). The European hypovirus, Hypovirus Cryphonectria hypovirus 1-80-2 (CHV1 80-2) was associated with an isolate collected from a field study established in Pocahontas County, WV where strains infected with Italian hypoviruses had been released. The second hypovirus, Hypovirus Cryphonectria hypovirus 3-CoLi 11-1 (CHV3 CoLi 11-1), is a hypovirus associated with an isolate recovered from a site at County Line, MI.

Isolates selected for this study were chosen based on their pigmentation and vegetative compatibility. Isolate pigmentation was the primary marker used to distinguish between isogenic virulent versus hypovirulent strains and between the brownpigmented hypovirus donor strain versus the orange-pigmented hypovirus receiver strain. The orange-pigmented YB-2 and brown-pigmented $80-2 \mathrm{C}$ were the two virulent isolates selected for use (Table 1). Isolate YB-2 was obtained from a canker at a study site in 
Marion County, West Virginia (M. Double) and was selected for use from the isolates recovered at that location because it was found to be consistently receptive to hypovirus acquisition when paired. The virulent isolate $80-2 \mathrm{C}$ was selected because of its brown pigmentation and its vegetative compatibility with isolate YB-2. For the purpose of this experiment, isolate $80-2 \mathrm{C}$ needed to be infected with $\mathrm{CHV} 3 \mathrm{CoLi} 11-1$. In all cases, the brown-pigmented strain $80-2 \mathrm{C}$ was the hypovirus-infected isolate used to deliver hypovirus to orange-pigmented virulent mycelium.

Table 1: Cryphonectria parasitica isolates and hypoviruses utilized throughout this project.

\begin{tabular}{|l|l|l|l|l|}
\hline Isolate & \multicolumn{1}{|c|}{ Hypovirus (Photograph of Isolate) } & Color of Cultures* & Growth** & \multicolumn{1}{c|}{ Source } \\
\hline YB-2 & None (Fig. 1-A) & Orange & Fast & Marion County, WV \\
\hline YB-2 & Hypovirus Cryphonectria hypovirus 1-80-2 (Fig. 1-B) & White (light orange) & Fast & Created for this project \\
\hline YB-2 & H. Cryphonectria hypovirus 3-Co-Li 11-1 (Fig. 1-C) & Orange & Slow & Created for this project \\
\hline 80-2C & None (Fig. 1-D) & Brown & Fast & Balbalian, 1998 \\
\hline 80-2C & H. Cryphonectria hypovirus 1-80-2 (Fig. 1-E) & White (light brown) & Fast & Balbalian, 1998 \\
\hline 80-2C & H. Cryphonectria hypovirus 3-Co-Li 11-1 Fig. 1-F) & Brown & Slow & Balbalian, 1999 \\
\hline
\end{tabular}

* Color of one-week-old colonies grown under bright light.

** Relative growth rate of colonies. 


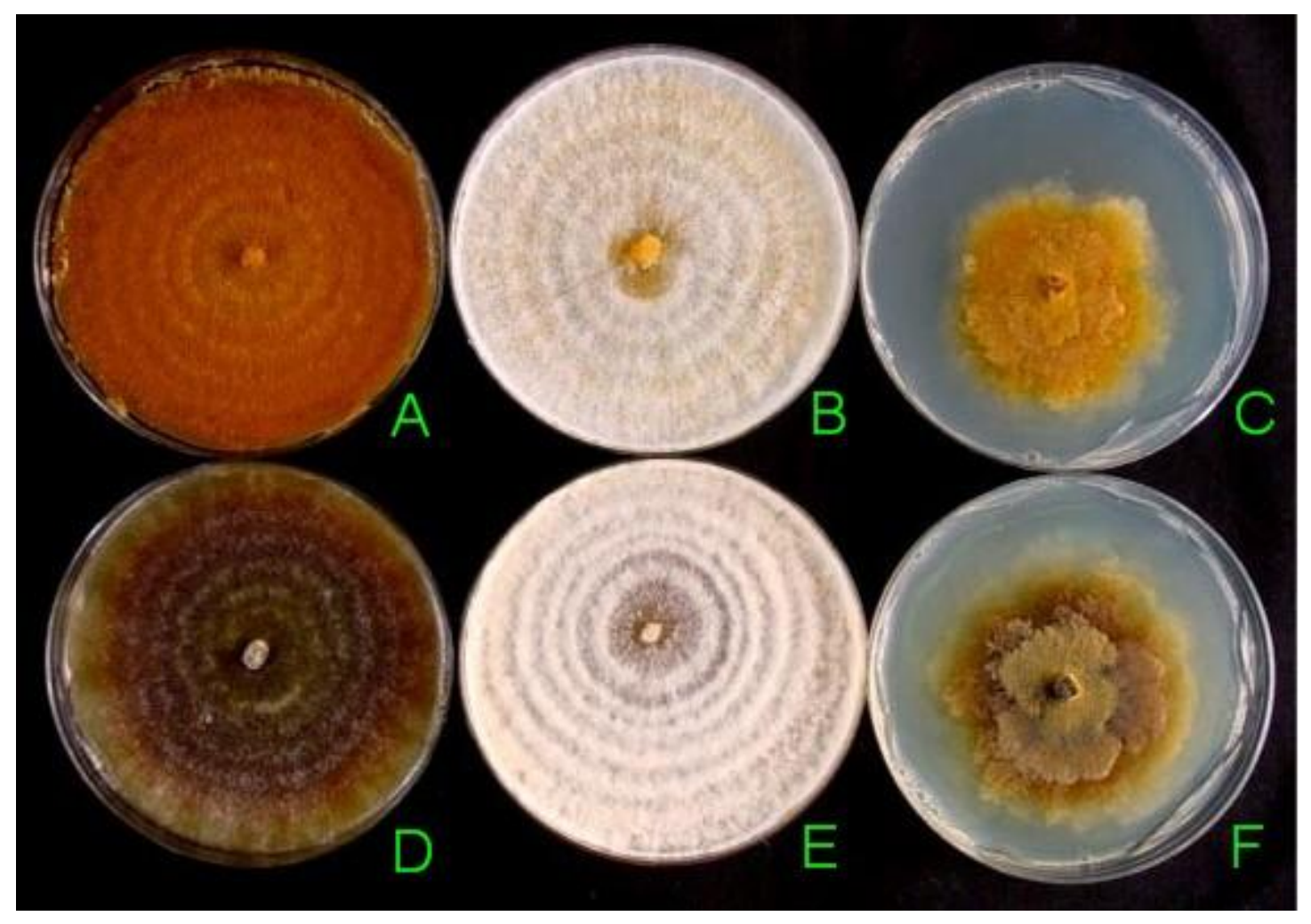

Figure 1: Isolates utilized throughout this study.
A. YB-2 (Orange-pigmented virulent)
B. YB-2 [CHV1 80-2]
C. YB-2 [CHV3 CoLi 11-1]
D. $80-2 \mathrm{C}$ (Brown-pigmented virulent)
E. 80-2C [CHV1 80-2]
F. 80-2C [CHV3 CoLi 11-1] 


\section{B. Verification of the assay for virulence phenotype}

This project required that isolate phenotype be scored as virulent or hypovirulent based on colony morphology and pigmentation. Compared with their uninfected standard cultures, isolates infected with Hypovirus Cryphonectria hypovirus 1-80-2 grew just as rapidly, appeared white and developed abundant aerial mycelia. Isolates infected with $H$. Cryphonectria hypovirus 3-CoLi 11-1 grew more slowly, retained their normal pigmentation but produced little to no aerial hyphae (Figure 1).

Independent verification of the hypovirus infection status (virulent-hypovirulent phenotype) of isolates YB-2 and 80-2C was conducted by Lynn Geletka at the University of Maryland using 24 isolates that were part of a pilot study set up and sampled similar to the rest of this experiment (Geletka, personal communication). Visualization of dsRNA was performed using Clamp R RT-PCR amplification (Kowalik, T.F., et al., 1990) of freeze-thaw method extracted DNA (Lecellier and Silar, 1994). Colonies infected with hypovirus yielded a strong band of approximately $270 \mathrm{bp}$ in length and no band of a similar size was exhibited from virulent colonies (Appendix A). Cultural morphology and pigmentation phenotype accurately predicted virulent or hypovirulent genotype in all 34 isolates that were tested. The methods used for the phenotypic verification are included as Appendix A because this study was not directly related to this master's project.

\section{Experiment I-A}

This experiment assessed the age at which Cryphonectria mycelium is no longer capable of acquiring hypovirus. This was done by monitoring the recovery of hypovirulent isolates over time. The experiment was started on June 8, 1999 with the initiation of 420 colonies of isolate YB-2 on potato dextrose agar (PDA) amended with antibiotics (Appendix B). Inoculum used to initiate colonies consisted of mycelial plugs taken from the leading edge of one-week-old cultures of YB-2. To meet the objective of the experiment, colonies were allowed to expand for $0,1,2,3,4,5$, or 6 weeks before hypovirus challenge. Next, and beginning one week after each challenge, unique sets of ten colonies were sampled weekly for six weeks. Each weekly sample was obtained 
using independently sampled colonies such that an individual colony was only sampled once.

Before challenge, colonies were maintained at $20^{\circ} \mathrm{C}$ and exposed to 16 hours of fluorescent light and 8 hours of dark per day. Growth was recorded weekly by tracing the colony margins directly on the bottom of the petri dishes using a fine-point permanent marker to form a pattern for sampling based on the expansion of each colony. Following hypovirus challenge, colonies were cultured at $20^{\circ} \mathrm{C}$ under total darkness and were not disturbed or traced until sampling.

Treatments consisted of three types of challenges to colonies that ranged in age from 0 - to 6-weeks-old. The three types of challenges were: 1) co-inoculations to zero week-old colonies; 2) colony margins of colonies that were one or two weeks-old; and 3) opposite of the colony initiation point of colonies that were three, four, five, and six weeks old (Figure 2). Each challenge to colonies ranging in age from zero to six weeks was replicated 60 times over each of the seven colony ages for a total of 420 colonies. The large number of replications was necessary because a colony was only sampled once because of the destructive sampling method, desiccation and contamination. Inoculum was placed with mycelium facing up using a scalpel. Co-inoculations consisted of mycelial plugs of strain 80-2C [CHV1 80-2] paired with plugs of YB-2 on day one of the experiment (week zero). Challenges after one or two weeks of growth were made with mycelial plugs of 80-2C [CHV1 80-2] at the colony leading edge. Placement of mycelial plugs of 80-2C [CHV1 80-2] after 3, 4, 5 and 6 weeks of growth for challenges required placement opposite of the colony initiation point (Figure 2). After challenge, plates were sealed with Parafilm ${ }^{\circledR}$ and maintained at $20^{\circ} \mathrm{C}$ with 24 hours per day of darkness as described above to allow for better hyphal anastomoses between the two different strains (M. Double, personal comm.).

Beginning one week after challenge, sets of ten colonies from each of the seven challenges were sampled weekly for six consecutive weeks. Samples consisted of mycelial plugs removed aseptically with a scalpel. Four samples, spaced as evenly as possible were removed from each of the traced weekly growth areas. This yielded 4,8 or 12 samples from each colony that had grown for one, two, three and more weeks, respectively. The only time 4 or 8 samples were taken was from co-inoculated colonies 
that were one- or two-weeks old at the time of sampling, respectively. More or less than 4 samples were taken from some irregular growth areas (e.g. lobate colonies), however, 12 samples total were still taken from those colonies. A record of where samples were taken from colonies was maintained by photocopying the plates immediately after challenge and by retaining the sampled plates until after colonies were scored for hypovirus infection (Figure 3). After about 3 weeks, records were maintained by hand drawings because so much fungal pigmentation was produced that it was impossible to distinguish features of the colonies on the copies.

Samples were cultured for one week under bright fluorescent light at room temperature. The resulting colonies were scored by comparing them to standards that were maintained throughout the course of the experiment. Colonies were scored as virulent or hypovirulent or contaminated.

Statistical comparisons between the frequency of OV and OHV isolates among the seven colony ages was performed using the parametric Tukey's Studentized Range Test for count and the nonparametric Wilcoxon Scores for Variable count t-tests within the SAS® statistical software package (SAS Institute Inc., Cary, NC). 


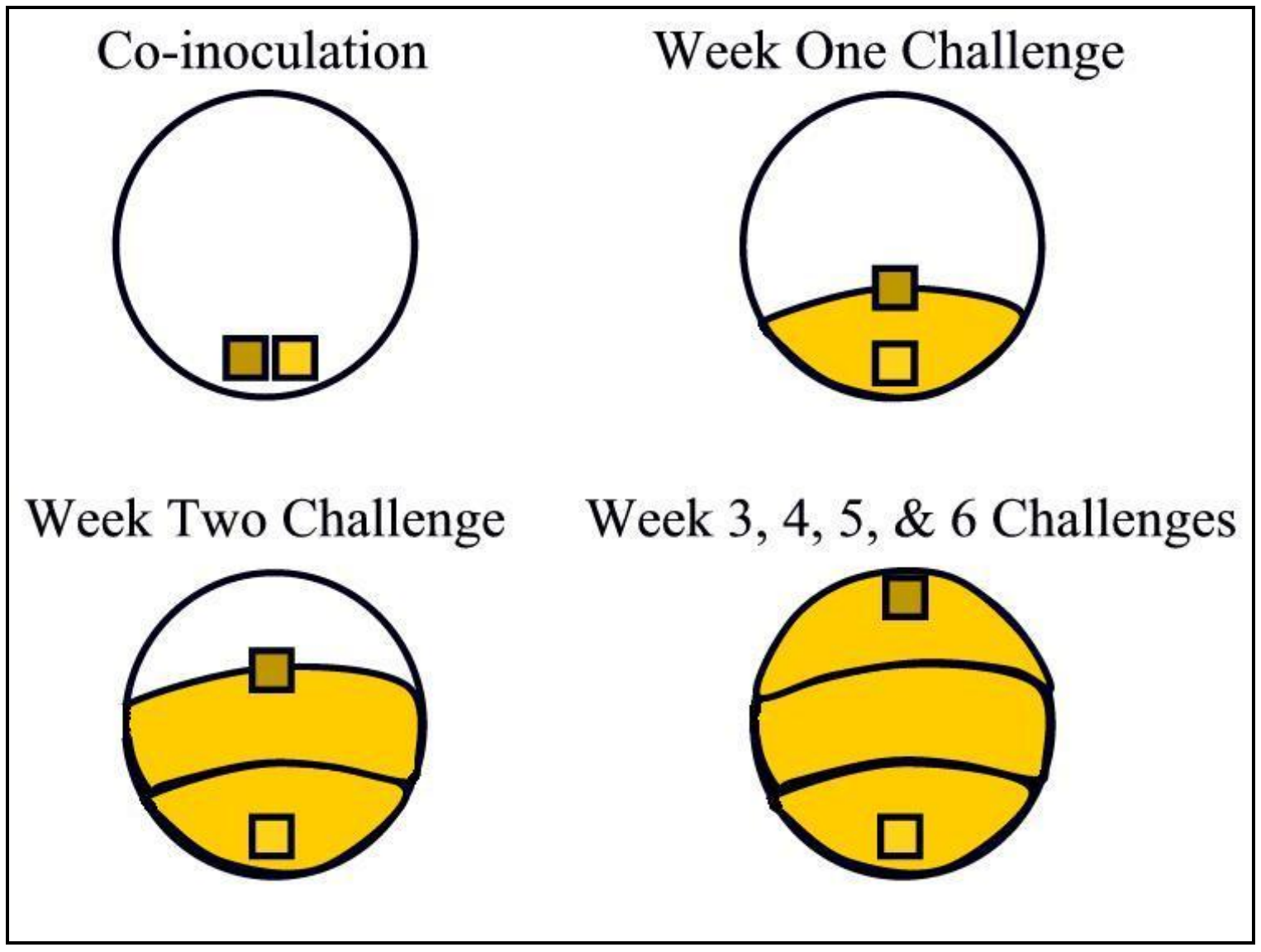

Figure 2: Cartoon depicting hypovirus challenge arrangements. [Challenges were made as co-inoculations, at the leading edge of expanding colonies or opposite of the colony initiation point. The orange square represents the colony initiation point and the brown square represents the hypovirus challenge point.] 


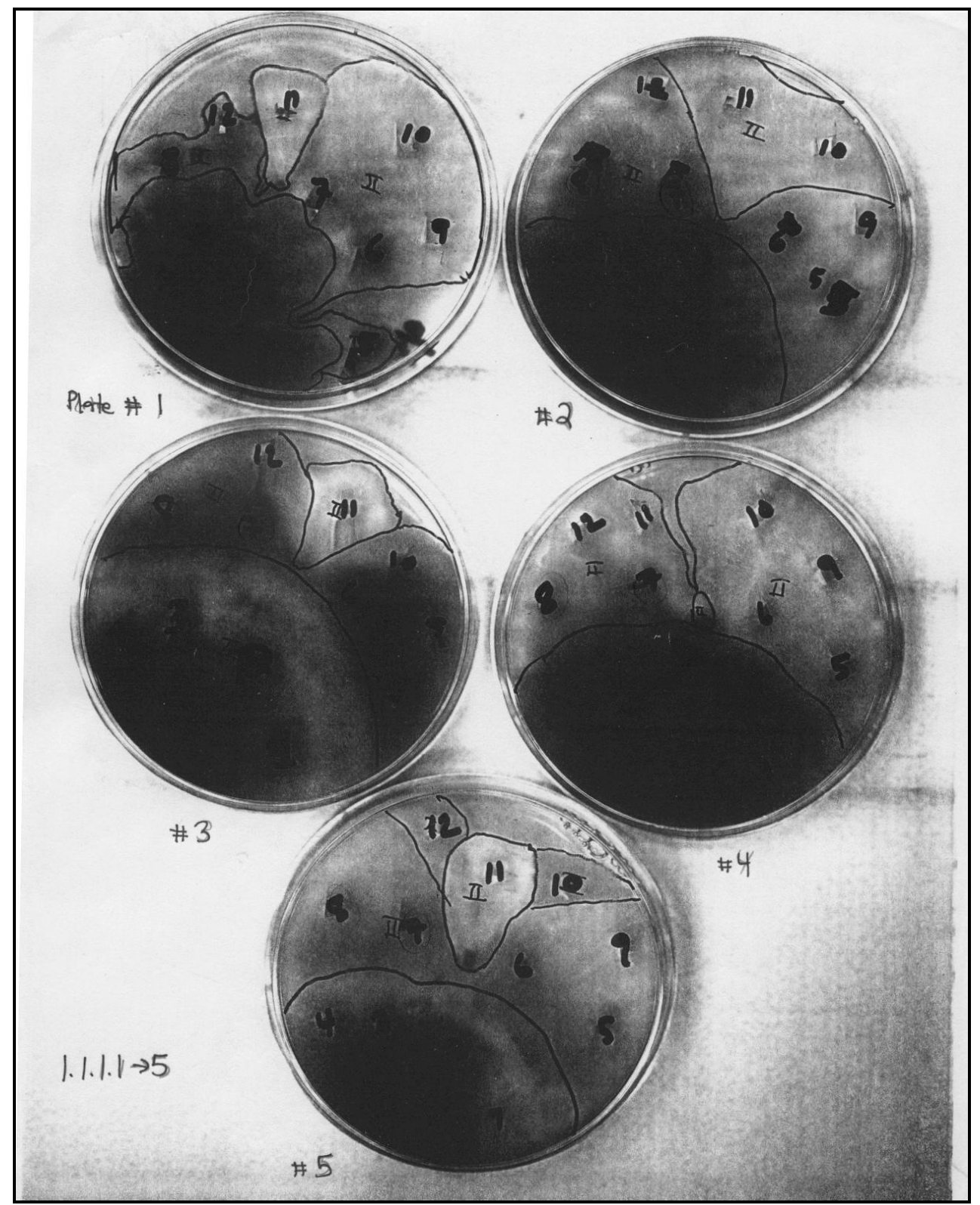

Figure 3: Example of the photocopy records maintained of plates after sampling. [Numbers written on the plates indicates the number of samples isolated from those colonies.] 


\section{Experiment I-B}

As with Experiment I-A, colonies of YB-2 were challenged weekly to monitor hypovirus transmission and colonization of established virulent mycelium. Experiment B began on June 18, 1999 with the initiation of 270 colonies of orange-pigmented isolate YB-2 on amended PDA (Appendix B). Three types of challenges (co-inoculations, challenges made to the leading edge of one-week-old colonies, or challenges made 0.5 $\mathrm{cm}$ behind the leading edge of one-week-old colonies) were made using three inoculum sources for a total of 9 treatments (Figure 4). Inoculum consisted of mycelial plugs of either isolate 80-2C infected with CHV1 80-2, isolate 80-2C infected with CHV3 CoLi 11-1 or 80-2C (virulent) as a control (Table 1). Each treatment contained 30 replications.

Immediately following initiation, co-inoculated colonies were cultured at $20^{\circ} \mathrm{C}$ under darkness until sampling, whereas colonies challenged after one week of growth were cultured at $20^{\circ} \mathrm{C}$ under 16 hours of fluorescent light per day prior to challenge. Following challenge, colonies challenged after one week of growth were cultured under darkness. Plates were kept sealed with Parafilm ${ }^{\circledR}$ to prevent contamination and desiccation. Colony growth was monitored weekly by tracing colony margins directly onto the bottom of the petri dishes using a permanent magic marker. By tracing colony expansion, a pattern for sampling was established.

Ten colonies from each treatment were sampled weekly for three weeks after challenge. Sampling was conducted as in Experiment A by taking 12 samples per colony using the pattern traced onto the petri dishes as a guide. The only exception to this was the first sample of co-inoculated cultures where only 5 samples ( 4 samples from the orange-pigmented mycelium and 1 sample from the brown-pigmented mycelium per plate) were taken per colony because of the small colony sizes. Records of sampling position were maintained as free-hand drawings of the colonies. Samples were cultured 4 per plate on PDA under continuous fluorescent lighting for one week at room temperature (Figure 5). Cultures were scored as virulent or hypovirulent based on morphology and pigmentation by comparing them to standards.

The proportion of OV and OHV isolates recovered per colony were compared using the parametric Tukey's Studentized Range Test for count and the non-parametric 
Wilcoxon Scores for Variable count t-tests with the SAS® statistical software package (SAS Institute Inc., Cary, NC).

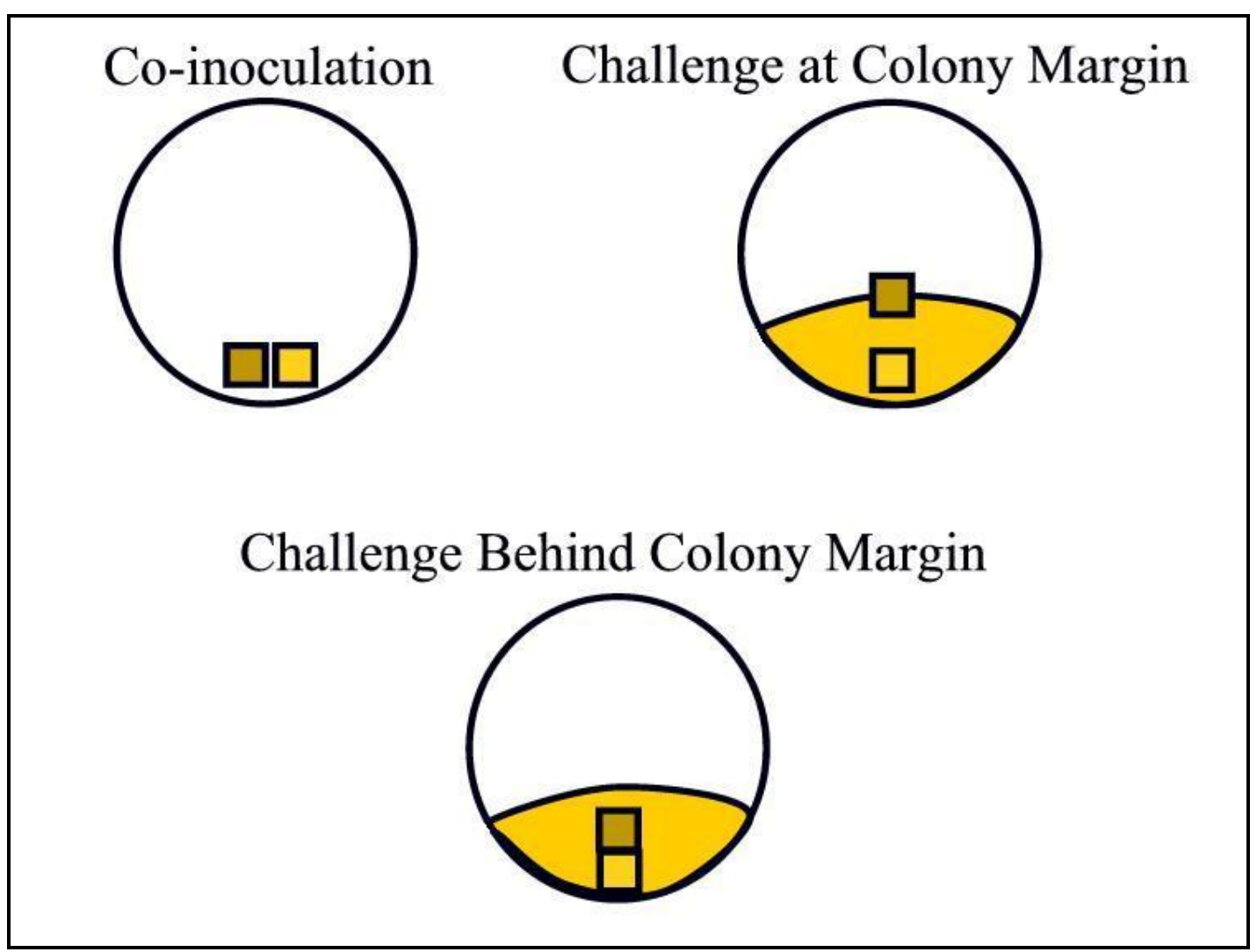

Figure 4: Cartoon depicting hypovirus challenges as conducted in Experiment IB. [Challenges consisted of co-inoculations, challenges to the colony leading edges, and challenges made behind colony leading edges.] 


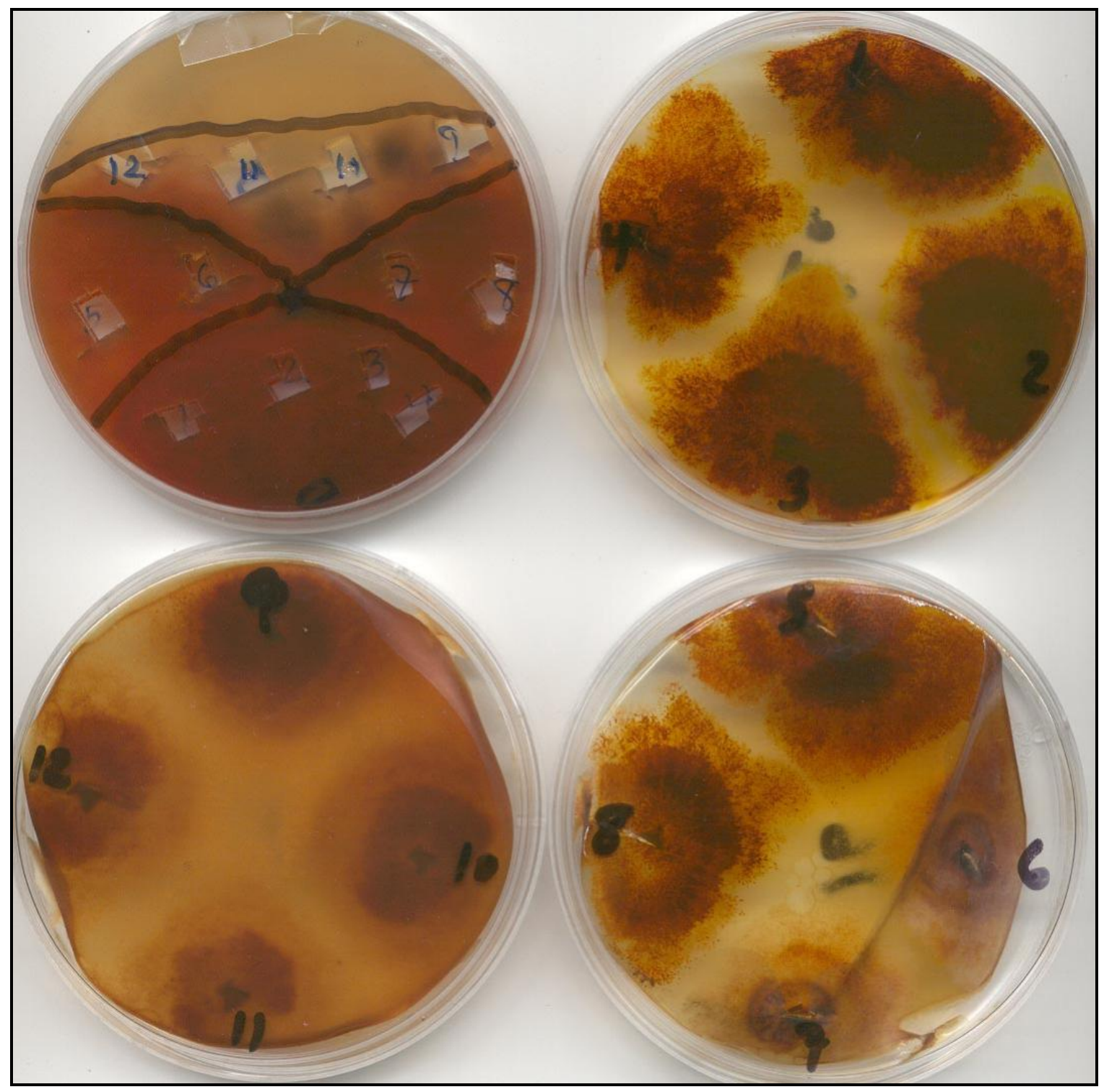

Figure 5: Example of the twelve isolates recovered from a one-week-old colony (upper left) challenged at the colony margin one week prior. [Note: samples 1-5 \& 8 are darkly pigmented (orange-pigmented virulent) and colonies do not fuse whereas samples 6, 7, 9-12 are lightly-pigmented (orange-pigmented hypovirulent) and colonies fused.] 


\section{CHAPTER I-ACQUISITION OF HYPOVIRUS IN VITRO RESULTS}

\section{Experiment I-A}

A total of 4,811 samples were obtained from 420 colonies over the six weeks of sampling. In total, 3,804 samples (79.1\%) were obtained from mycelium that had extended prior to hypovirus challenge and 1,007 samples (20.9\%) were obtained from mycelium that had expanded after challenge. The majority of the isolates $(4,145$ or $86 \%)$ were scored as orange-pigmented virulent (OV) (Table 2). A total of 588 orangepigmented hypovirulent $(\mathrm{OHV})$ isolates $(12.2 \%)$ also were recovered. The remaining isolates, including brown-pigmented isolates were scored as contaminants (C). Over $99 \%$ of the OHV isolates were obtained from sampling of mycelium that had expanded after challenge. Samples obtained from mycelium that extended before challenge only yielded two cultures $(0.3 \%)$ that were scored OHV. All brown-pigmented hypovirulent isolates were recovered from samples of mycelium that had extended after challenge.

The results from the parametric and nonparametric t-tests were similar and the frequency of hypovirulent isolates obtained per colony had a normal distribution, therefore only the results from the parametric comparisons are described. The proportion of orange-pigmented hypovirulent isolates obtained per colony from mycelium that extended after the point of challenge was significantly greater $\left(\mathrm{R}^{2}=0.6228, \mathrm{P}<0.0001\right)$ than from isolations from mycelium that extended prior to challenge. Conversely, significantly more orange-pigmented virulent isolates were obtained per colony from sampling of mycelium established prior to challenge $\left(\mathrm{R}^{2}=0.7056, \mathrm{P}<0.0001\right)$. Hypovirulent isolates almost never were obtained from mycelium that had expanded prior to challenge (Table 2). 
Table 2: The total number of orange-pigmented virulent $(\mathrm{OV})$, orange-pigmented hypovirulent (OHV), brown-pigmented hypovirulent (BRHV), and contaminated (C) isolates recovered from mycelium that was established from 6 weeks before (-) to 3 weeks after $(+)$ challenge.

Mycelium established in \# of Weeks prior to challenges

\section{Mycelium established} in \# of

\begin{tabular}{|c|c|c|c|c|c|c|c|c|c|c|c|c|}
\hline & $\underline{-6}$ & $\underline{-5}$ & $-\underline{-4}$ & $\underline{-3}$ & $\underline{-2}$ & $\underline{-1}$ & & +1 & +2 & +3 & & \\
\hline $\begin{array}{c}\text { Isolate } \\
\text { Recovered }\end{array}$ & & & & & & & $\begin{array}{l}\text { Sub- } \\
\text { Total }\end{array}$ & & & & $\begin{array}{l}\text { Sub- } \\
\text { Total }\end{array}$ & Sum \\
\hline OV & 235 & 671 & 716 & 708 & 869 & 561 & 3,760 & 344 & 37 & 4 & 385 & 4,145 \\
\hline OHV & 0 & 0 & 0 & 0 & 0 & 2 & 2 & 318 & 204 & 64 & 586 & 588 \\
\hline BRHV & 0 & 0 & 0 & 0 & 0 & 0 & 0 & 29 & 8 & 0 & 37 & 37 \\
\hline $\mathbf{C}$ & 5 & 51 & 0 & 0 & 0 & 0 & 56 & 0 & 0 & 0 & 0 & 56 \\
\hline
\end{tabular}




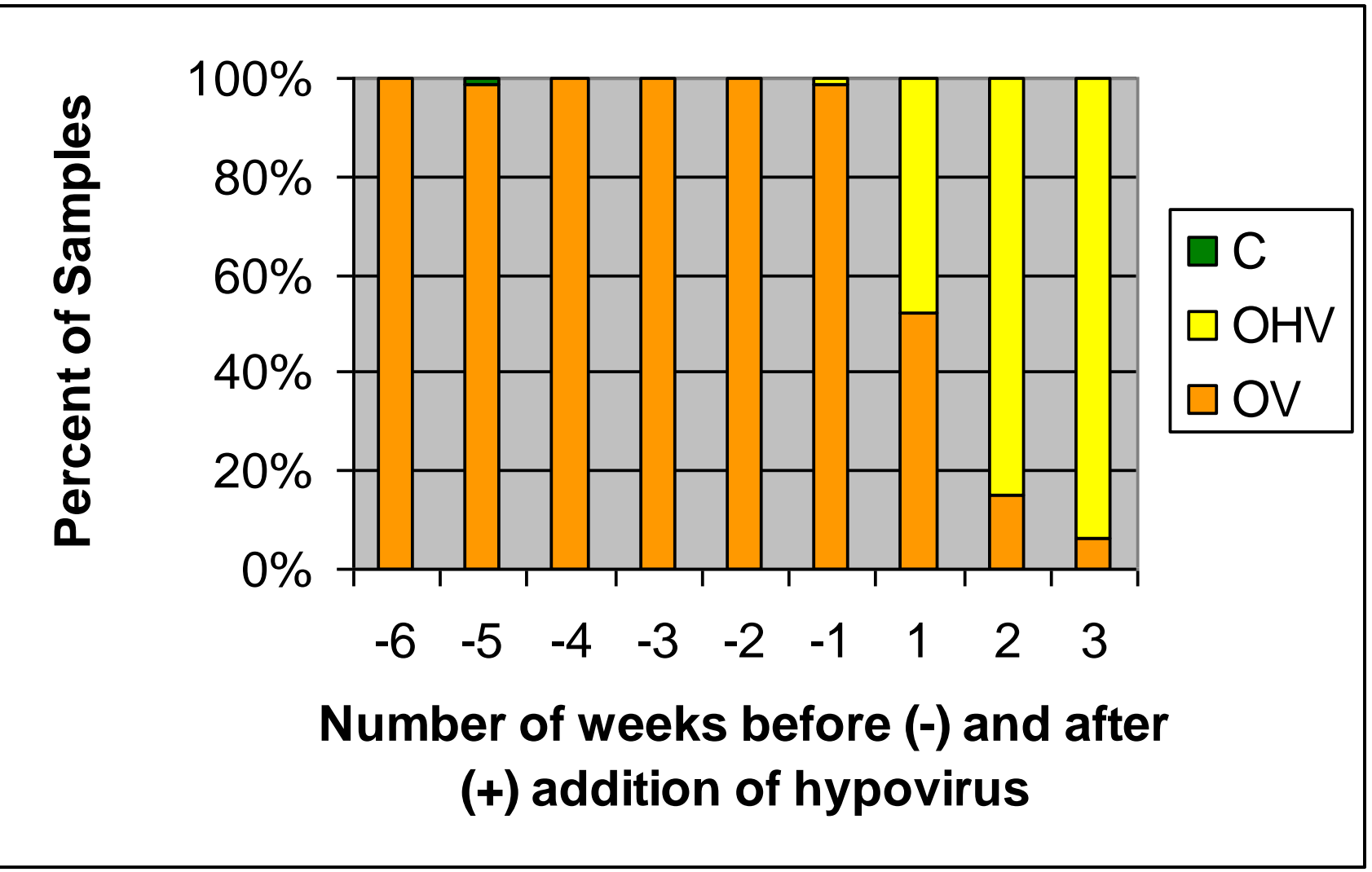

Figure 6: Percent orange-pigmented virulent $(\mathrm{OV})$, orange-pigmented hypovirulent $(\mathrm{OHV})$ and contaminated $(\mathrm{C})$ isolates recovered from mycelium that had expanded from 1-to-6 weeks before (-) to 1-to-3 weeks after (+) challenge. 
Challenging virulent 0-to-6-week-old YB-2 colonies with the hypovirulent strain 80-2C [CHV1-80-2] required three different mycelium and agar plug arrangements: coinoculations of zero week-old colonies, challenges made at colony leading edge of oneand two-week-old colonies, and challenges behind the colony leading edge on those three or more week-old colonies that had fully expanded within the petri dish. The mean proportion of $\mathrm{OV}$ and $\mathrm{OHV}$ isolates recovered per colony from each of the three challenge-inoculum arrangements were significantly different $(\mathrm{P}<0.0001)$.

From the 60 colonies that were co-inoculated with isolate YB-2 and 80-2C [CHV1-80-2] on week zero, sets of ten colonies were sampled weekly for six weeks. In total, $427 \mathrm{OHV}, 94 \mathrm{OV}$ and 7 contaminated isolates were cultured. A mean of 7.1 OHV isolates out of $8.8(80.9 \%)$ total isolations per colony were obtained from co-inoculated colonies (Figure 7). An average of 1.6 isolates per colony was scored OV. Compared with the other two mycelium and agar plug arrangements for challenge, samples taken from co-inoculated plates had the greatest mean number of OHV isolates recovered per colony over the six weeks of sampling.

Colonies challenged at the leading edge after one or two weeks of growth yielded significantly $(\mathrm{P}<0.0001)$ fewer orange-pigmented hypovirulent isolates per colony than co-inoculated colonies on week zero. A total of 1,199 isolations were taken from 60 each of 120 YB-2 colonies challenged at the colony leading edge with 80-2C [CHV1 80-2] after one or two weeks of growth, and then sampled weekly for six weeks. In total, 995 of the isolates were scored as OV (83.0\%), 174 isolates were scored as OHV (14.5\%), and 30 isolates were scored as contaminants (2.5\%). On average, 10.1 samples were taken per colony, with 8.3 samples scored OV, 1.5 samples scored OHV and 0.3 samples scored C per colony.

Only two hypovirulent isolates were recovered from mycelium that was established prior to hypovirus challenges, and both of those isolates came from one colony that was challenged after one week and sampled one week later. The remaining 98.9\% of the $\mathrm{OHV}$ isolates were recovered from mycelium that expanded after challenge.

No significant differences were detected in the proportion of OV or OHV isolates recovered from mycelium that had expanded one week before or one week after challenge between colonies that were one- or two-weeks-old at the time of challenge. 
Therefore, the data from challenges to one- and two-week-old colonies were pooled. Samples taken from one-week-old colonies yielded a mean and standard deviation of $18.3 \pm 6.8 \%$ orange-pigmented hypovirulent samples after six weeks of sampling (Figure 8). Samples from colonies challenged at the leading edge of two-week-old colonies yielded a mean and standard deviation of $5.7 \pm 5.1 \%$ orange-pigmented hypovirulent samples (Figure 9).

Colonies that were allowed to expand for three or more weeks prior to challenge completely filled the petri dishes by the time of challenge. Hypovirulent isolates were never recovered from mycelium that had expanded three or more weeks prior to challenge and therefore no significant differences were detected (data not shown). A total of 3,084 samples were taken from 240 colonies. 3,028 (98.2\%) of the isolates recovered from mycelium that had expanded from 3-6 weeks before challenge were scored OV. Significantly greater $(\mathrm{P}<0.0001)$ orange-pigmented virulent isolates were recovered from colonies challenged opposite of the initiation point than from coinoculated colonies (week zero) or challenges to colony leading edges (weeks one and two). Fifty-six isolates (1.8\%) were scored as contaminants. All of the contaminants were recovered from mycelium that was five or six weeks old at the time of challenge and were the oldest colonies in the study (Table 2).

All but two hypovirulent isolates were recovered from mycelium that extended following hypovirus challenge and virulent mycelium that extended following challenge continued to yield virulent isolates (Figure 5). 


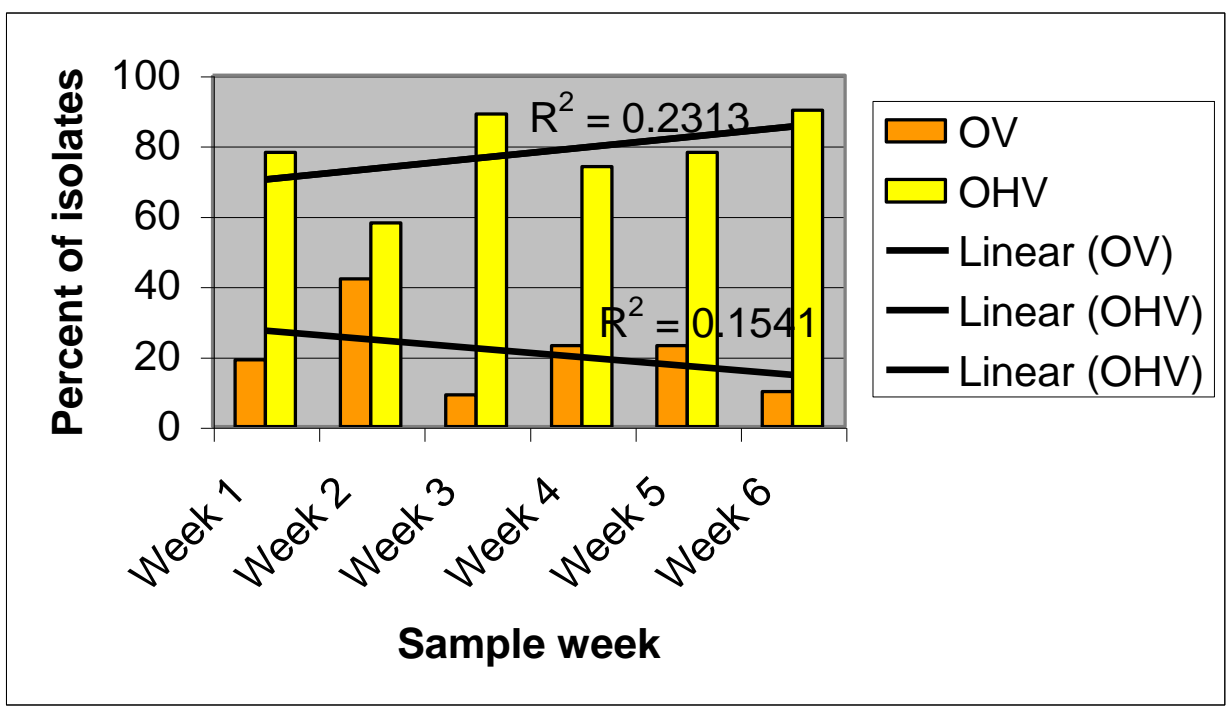

Figure 7: Percent and linear trends of orange-pigmented virulent $(\mathrm{OV})$ and hypovirulent $(\mathrm{OHV})$ isolates recovered from co-inoculated colonies of YB-2 and 80-2C [CHV1 80-2] over six weeks of sampling.

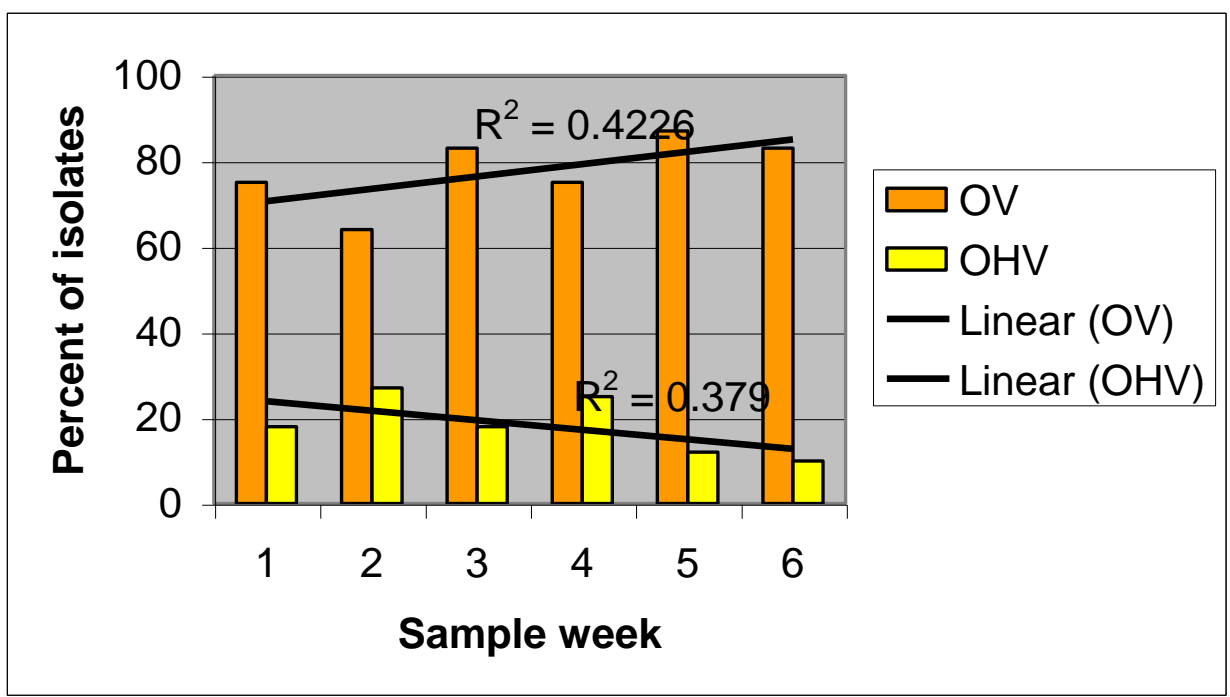

Figure 8: Percentage and linear trends of orange-pigmented virulent (OV) and orange-pigmented hypovirulent $(\mathrm{OHV})$ isolates recovered from one-week-old colonies challenged at the colony leading edge over six weeks of sampling. 


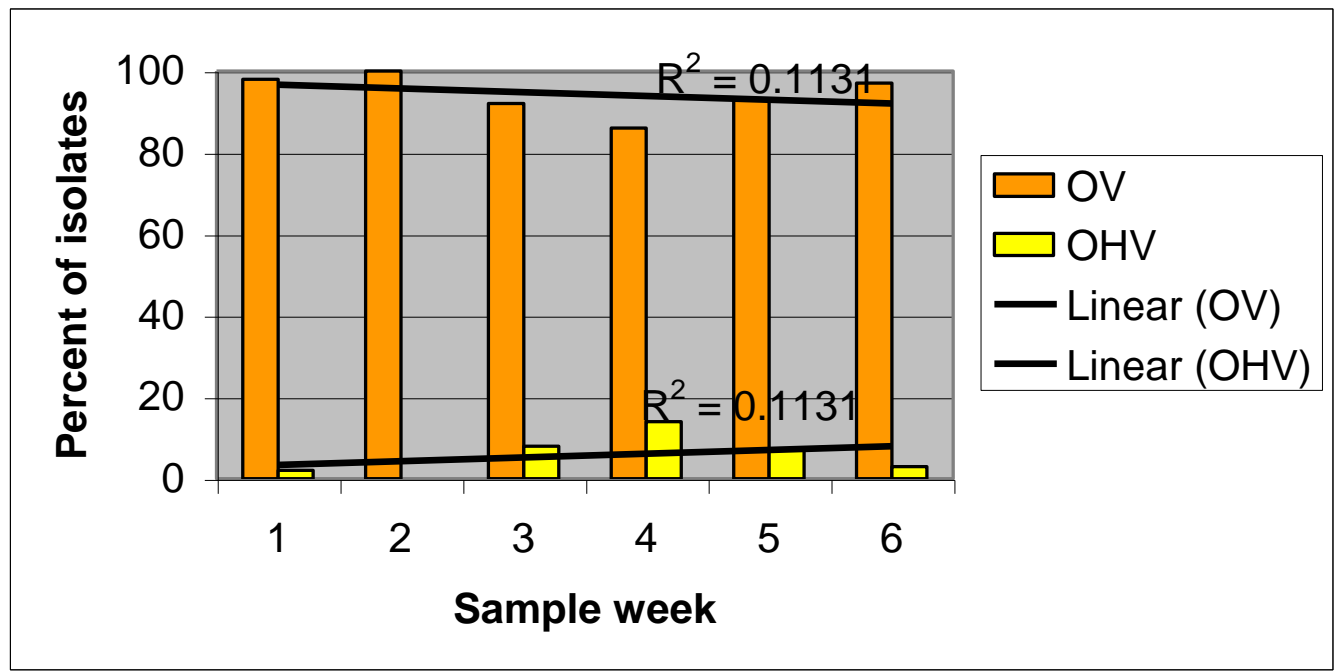

Figure 9: Percentage and linear trends of orange-pigmented virulent and orangepigmented hypovirulent isolates recovered from two-week-old colonies challenged at the colony leading edge over six weeks of sampling. 


\section{Experiment I-B}

Each challenge type consisted of 30 replicate colonies (270 total colonies), with 10 colonies sampled weekly for three weeks beginning one week after challenge. In total, 2,890 isolations were obtained. A highly significant difference (Tukey's: $\mathrm{R}^{2}=0.7954, \mathrm{P}<0.0001$ and Wilcoxon: Chi-square $\left.=411.7613, \mathrm{P}<0.0001\right)$ was found between the proportion of $\mathrm{OV}$ and $\mathrm{OHV}$ isolates between colonies challenged with hypovirulent inoculum versus controls.

Of the 60 YB-2 colonies that were co-inoculated with hypovirulent (HV) strains: $\mathrm{HV}$ isolates were obtained from 47 colonies that faced an HV-inoculum challenge (78.3\% transmission of hypovirus), no HV strains were isolated from eight colonies (13.3\%), and five colonies were lost due to contamination (8.3\%). Co-inoculations made with isolate 80-2C [CHV1 80-2] yielded $98 \mathrm{OV}$ and $62 \mathrm{OHV}$ isolates (Figure 10). Coinoculations made with isolate 80-2C [CHV3 CoLi 11-1] yielded 95 OV and $173 \mathrm{OHV}$ isolates (Figure 10). Significantly more OHV isolates were obtained from colonies challenged with the CHV3-type versus the CHV1-type hypovirus.

From 30 colonies co-inoculated with BRV as a control, 216 OV and 74 BRV isolates were recovered following the three samples. $\mathrm{HV}$ isolates were never isolated from controls on any sample date and recovery of OV isolates from colonies was significantly greater $(\mathrm{P}<0.0001)$ than from colonies co-inoculated with hypovirulent mycelium.

Of the 60 colonies that were challenged at the colony leading edge with hypovirulent inoculum: $\mathrm{HV}$ isolates were isolated from 44 colonies $(73.3 \%$ transmission of hypovirus), HV strains were not isolated from 13 colonies (21.7\%), and 3 colonies were lost due to contamination (5\%). Challenges made at the colony leading edge with 80-2C [CHV1 80-2] yielded $192 \mathrm{OV}$ and $143 \mathrm{OHV}$ isolates (Figure 10). Challenges made at the colony leading edge with 80-2C [CHV3 CoLi 11-1] yielded $242 \mathrm{OV}$ and 82 OHV isolates (Figure 10). Percent transmission of hypovirus was similar for both hypoviruses when colonies were challenged at the leading edge (Figure 10).

Colonies challenged at colony leading edge with BRV inoculum as controls yielded $360 \mathrm{OV}, 11 \mathrm{BRV}$, and one contaminated isolates. No hypovirulent isolates were obtained from sampling of controls. Significantly greater $(\mathrm{P}<0.0001) \mathrm{OV}$ isolates were 
obtained from colonies challenged with BRV than with either the CHV1-type or CHV3type hypovirus.

Of the 60 colonies that were challenged behind the colony margin with hypovirulent strains: HV isolates were recovered from 7 colonies $(11.7 \%$ transmission rate), no $\mathrm{HV}$ isolates were recovered from 50 colonies $(83.3 \%)$, and 3 colonies were lost due to contamination (5\%). Colonies challenged behind the leading edge with $80-2 \mathrm{C}$ [CHV1 80-2] yielded $334 \mathrm{OV}, 22 \mathrm{OHV}$, and 4 contaminated isolates (Figure 10). Two of the $22 \mathrm{OHV}$ isolates were obtained from mycelium established prior to challenge. Challenges made with 80-2C [CHV3 CoLi 11-1] yielded $322 \mathrm{OV}, 14 \mathrm{OHV}$, and 0 contaminated isolates (Figure 10). Eight of the $14 \mathrm{OHV}$ isolates were obtained from mycelium established prior to hypovirulent inoculum challenges. No significant differences were found between colonies challenged behind colony margins with either CHV1 or CHV3 hypovirus or between challenges made behind the leading edge with both hypoviruses and the control. When colonies were challenged behind the leading edge, some orange-pigmented virulent samples were obtained between the challenge point and newly extending hypovirulent mycelium at the colony leading edge.

Colonies challenged with inoculum of $80-2 \mathrm{C}$ behind the leading edge yielded 340 $\mathrm{OV}$ isolates and 8 contaminants. No hypovirulent isolates were obtained from colonies challenged behind the colony margin as controls. 


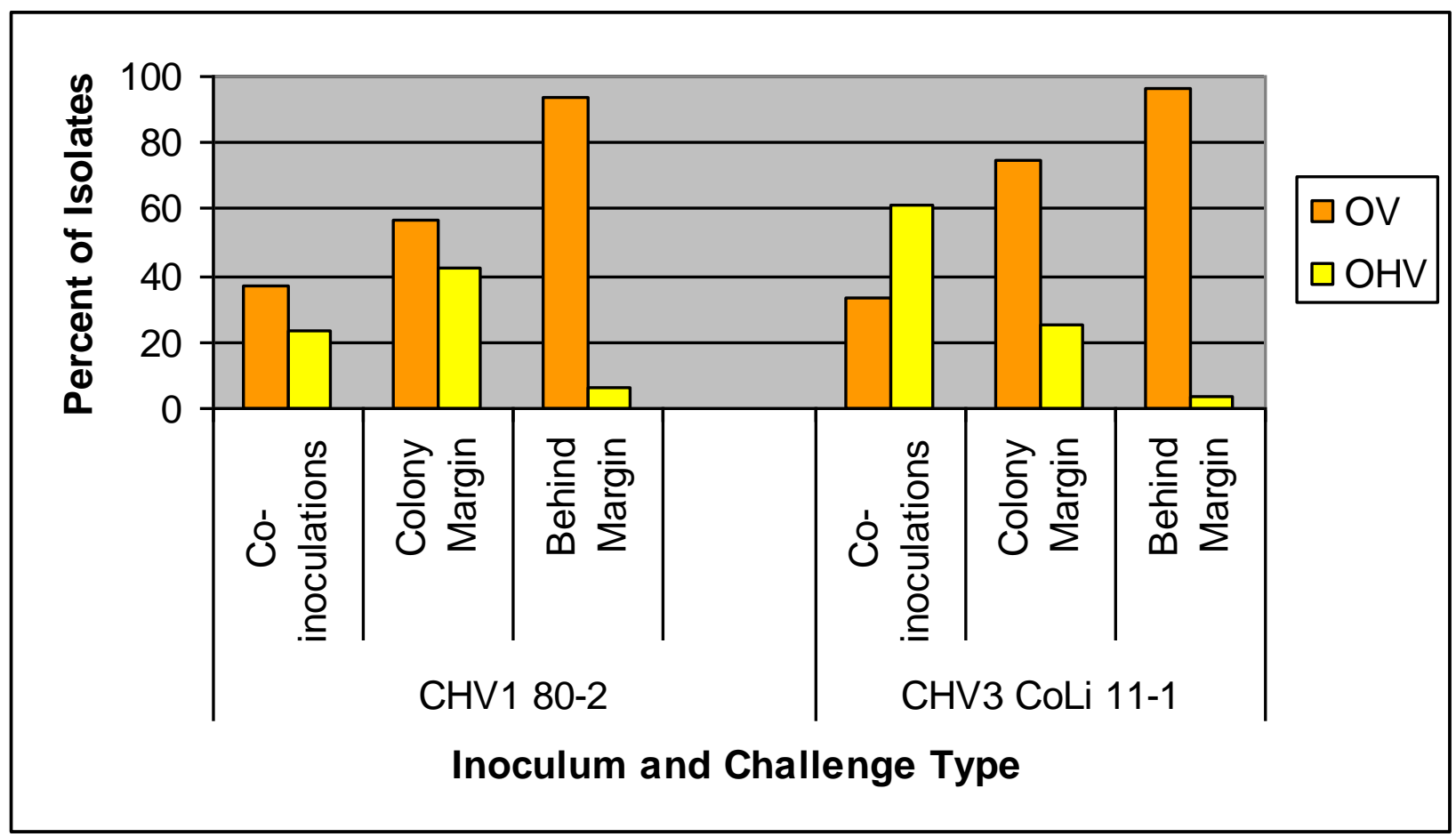

Figure 10: Summary of data from three weeks of sampling of the percent orangepigmented virulent and orange-pigmented hypovirulent isolates recovered from colonies when hypovirulent inoculum challenges were made as co-inoculations ( 0 weeks old), challenge to colony margins ( 1 week old), and challenges behind colony margins (1 week old). 


\section{CHAPTER II-ACQUISITION OF HYPOVIRUS IN VIVO METHODS AND MATERIALS}

This experiment compared the recovery of hypovirulent and virulent $C$. parasitica isolates cultured from bark samples taken from regions of cankers formed before and after introduction of a hypovirulent isolate. The change in recovery of hypovirulent isolates was monitored over time.

Healthy Castanea dentata stems were selected from a xeric oak-maple-chestnut mixed hardwood stand that had previously been defoliated by gypsy moth larvae. The severe damage to the canopy released the chestnuts from the understory. The stand was located on Meadow Mountain in Forest Management Compartment 25 of the Savage River State Forest in Garrett County, Maryland. Access to the plots was by a state forest maintenance road located adjacent to Lower New Germany Road. The diameter at breast height (DBH) of all trees was measured with a diameter-tape and bark thickness was measured with a bark gauge.

On June 25, 1998, the orange-pigmented virulent Cryphonectria parasitica isolate YB-2 (Table 1) was used to initiate 4 cankers on each of 10 trees. Cankers were spaced as equally as possible around the stems as allowed for by the trees' branching habits and adjacent stems at $0.5,1.0,1.5$ and $2.0 \mathrm{~m}$ off the ground. Inoculation points were made by wounding trees to the xylem using a 5/16" diameter leather punch. Inoculum plugs were made using the same leather punches to cut mycelium from the margins of one-week-old colonies of isolate YB-2. After inoculation, wounds containing the mycelial plugs were covered with masking tape to retain the inoculum in the wound holes and retard drying.

Following initiation, canker expansion was monitored monthly by tracing canker margins directly on the bark with a black permanent marker. Margins were traced to maintain a record of canker expansion over time and the traced regions served to designate areas for sampling. Cankers that did not visibly expand were not traced. Tracings occurred at 7 dates: 7/31/98, 8/24/98, 9/16/98, 10/16/98, 5/25/99, 6/25/99, and 10/29/99. After tracing, canker length and width $(\mathrm{cm})$ were measured.

Cankers were challenged with hypovirus on October 21, 1998 after allowing for

four months of expansion. Challenges consisted of inoculating wounds created using the 
same procedure that was used for canker initiation. Wounds were made to canker margins at lowest point on each canker. Inoculum consisted of mycelial plugs of isolate 80-2C infected with HV 1-80-2 (Table 1). Controls were challenged with mycelial plugs of the virulent isolate 80-2C. In total, 24 cankers on 6 trees were challenged with strain 80-2C infected with HV 1-80-2 and 16 cankers on 4 trees were challenged as controls. All 4 cankers on a single tree were challenged with the same inoculum type. Trees were randomly assigned virulent or hypovirulent inoculum using dice after sorting the trees into groups to ensure there were thin and thick barked control and test trees.

One month after challenge, cankers were sampled to obtain bark plugs to culture C. parasitica. Subsequent samples were taken on 12/21/98, 1/24/99, 2/26/99, 3/26/99, $4 / 26 / 99$, and 5/25/99 6/25/99, 11/25/99 and 2/25/00. Four samples were removed from each of the traced growth regions using a 2-mm bone marrow biopsy tool (Figure 11). Sampling locations from each growth region were at the 12:00, 3:00, 6:00 and 9:00 positions. Due to the small size of canker centers, the first traced growth region was not sampled on the second and third samplings to prevent the region from being destroyed. To keep bark plugs samples organized and prevent their loss, they were placed in 96-well microtiter plates (Figure 11) and held down with tape. Plates were then returned to the laboratory and immediately frozen.

After removing the tape used to hold the plugs in place, a wire mesh screen, held in place with rubber bands, was used to keep the bark plugs from floating out of the microtiter wells during sterilization. After sterilization, plates were rinsed with $0.5 \mathrm{~L}$ of autoclaved Milli-Q® water. Bark plugs were then surface sterilized for 13 minutes by soaking the entire microtiter plate in $10 \%$ sodium hypochlorite (bleach), $0.45 \mathrm{~L}$ autoclaved Milli-Q® ${ }^{\circledR}$ water, and 10 drops of liquid soap to reduce surface tension. $C$. parasitica was cultured by first aseptically removing the bark plugs from the microtiter plates with sterile forceps then placing them on glucose yeast extract (GYE) agar in 100mm-diameter petri dishes (Appendix A). The 4 samples from each traced region were cultured on a single dish of GYE.

Colonies were incubated for 3 to 4 days under 24 hours of bright fluorescent lighting at room temperature. Colonies of $C$. parasitica that developed were transferred to $100-\mathrm{mm}$-diameter petri dishes containing potato dextrose agar (PDA) and grown under 
continuous lighting for one week on PDA (Appendix A). Resulting colonies were scored for hypovirus infection based on their morphology and pigmentation by comparing them to standard cultures that were maintained throughout the course of the study.

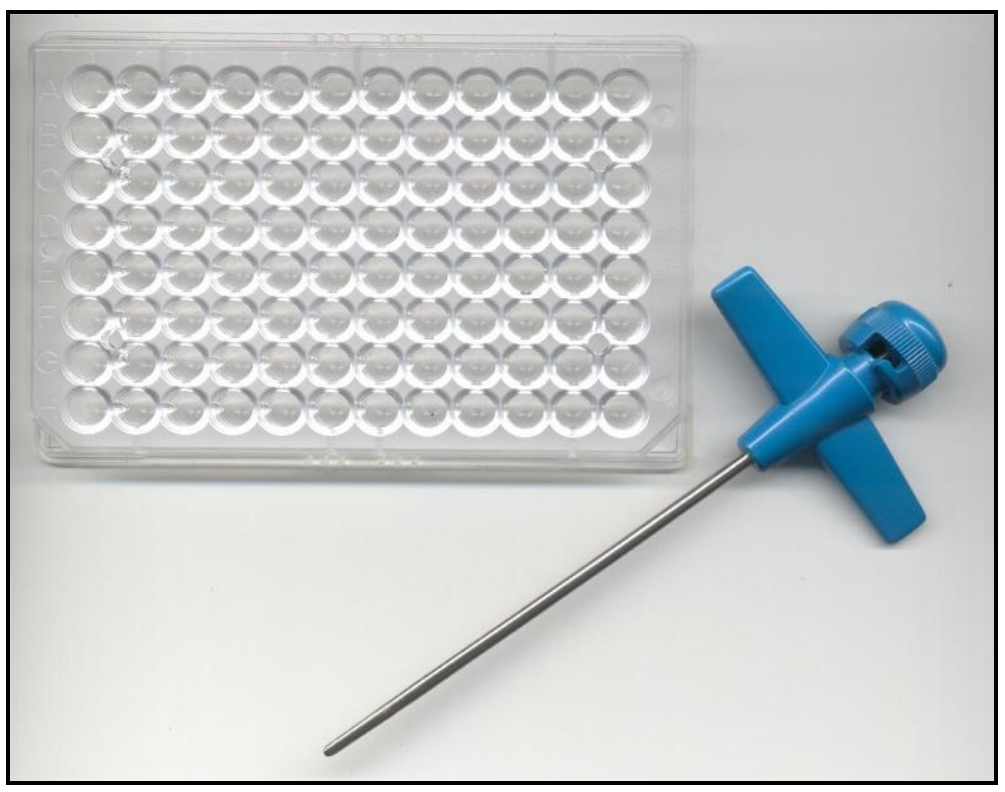

Figure 11: Bone marrow biopsy tool and microtiter dish utilized to obtain and transport bark plug samples to the laboratory, respectively. 


\section{RESULTS}

\section{A. American chestnut stems}

The American chestnuts selected for this study were apparently healthy trees that had been released from the understory due to previous gypsy moth defoliation of the oak overstory. Diameter at breast height (DBH) ranged from 7.5 to $19.1 \mathrm{~cm}$ with an average and standard deviation of $11.3 \pm 4.4 \mathrm{~cm}$ (Table 3). Bark thickness ranged from 1.27 to $6.35 \mathrm{~mm}$ with an average and standard deviation of $3.68 \pm 1.94 \mathrm{~mm}$ (Table 3). Thinbarked trees (bark thickness 1.27 to $2.54 \mathrm{~mm}$ ) tended to have a smooth gray-green appearance and lacked dead outer bark. Thick-barked trees (bark thickness $3.81 \mathrm{~mm}$ or greater) tended to have a rough, gray-colored appearance and possessed outer bark. Bark thickness was strongly correlated with DBH; trees with larger DBH had thicker bark ( $>0.0085$ ). No correlations between bark thickness and recovery of hypovirulent isolates were made.

Table 3: Diameter at breast height (DBH) and bark thickness and rating of trees used for this study $\left({ }^{*}\right.$ Cankers on these trees were not treated with a hypovirulent strain and served as controls).

\begin{tabular}{|c|c|c|c|}
\hline $\begin{array}{c}\text { Tree } \\
\text { Designation \# }\end{array}$ & $\underline{\text { DBH }(\mathbf{c m})}$ & Bark Thickness $(\mathbf{m m})$ & Bark Rating \\
\hline $3^{*}$ & 7.7 & 2.54 & Thin \\
\hline $7^{*}$ & 7.5 & 5.08 & Thick \\
\hline $11^{*}$ & 8.1 & 1.27 & Thin \\
\hline $15^{*}$ & 19.1 & 6.35 & Thick \\
\hline 16 & 12.5 & 2.54 & Thin \\
\hline 17 & 16.8 & 6.35 & Thick \\
\hline 21 & 7.8 & 2.54 & Thin \\
\hline 23 & 15.5 & 5.08 & Thick \\
\hline 25 & 10.4 & 3.81 & Thick \\
\hline 26 & 7.5 & 1.27 & Thin \\
\hline Mean & $\mathbf{1 1 . 3}$ & $\mathbf{3 . 6 8}$ & \\
\hline
\end{tabular}




\section{B. Canker expansion}

Although cankers were monitored monthly after initiation, they were traced and measured only when the radial expansion of the majority of the cankers was sufficient to allow for sampling (length of expansion $\sim 1.0 \mathrm{~cm}$ ). This resulted in seven dates at which cankers could be traced and measured. However, since not all of the cankers expanded equally, some were not traced; these were recorded as "no new growth" and were not remeasured. All four cankers on the thick-barked control tree 7 never visibly expanded during the course of the study, therefore, length and width data was not possible to obtain for these cankers. Length and width data were omitted from the data set presented in Tables 4-5. Average length and width $(\mathrm{cm})$ of test and control cankers is listed in Table 4. Cankers did not appear to expand from November 1998 until late in the following spring, with new growth recorded the last week of May 1999. In general, the length and width of test cankers was equal or less than those of control cankers but differences were not significant (Table 4).

Canker area was estimated from length and width data using the formula for the area of an ellipse $\left(\right.$ Area $_{\text {ellipse }}=\pi *($ length*width/4)). Mean canker areas are listed in Table 5. Mean canker area of cankers treated with hypovirulent inoculum increased from 22.1 to $925.6 \mathrm{~cm}^{2}$ between the first and last measurement dates, respectively. No significant differences were found between treated and control canker area expansion until the last measurement date (Table 5). 
Table 4: Average canker length and width $(\mathrm{cm})$ of cankers treated with hypovirulent (HV) versus virulent (V) inoculum.

\begin{tabular}{|c|c|c|c|c|}
\hline & \begin{tabular}{|c|} 
HV- \\
Inoculated \\
Cankers \\
\end{tabular} & & \begin{tabular}{|c|} 
BRV- \\
Inoculated \\
Cankers \\
\end{tabular} & \\
\hline & Length & Width & Length & Width \\
\hline Jul-98 & 3.6 & 1.7 & 4.5 & 1.8 \\
\hline Aug-98 & 6.2 & 3.2 & 6.8 & 3.5 \\
\hline Sep-98 & 8.9 & 5.2 & 9.5 & 5.5 \\
\hline Oct-98 & 11.3 & 7.3 & 12.1 & 6.9 \\
\hline May-99 & 16.1 & 11.1 & 16.1 & 11.1 \\
\hline Jun-99 & 17.7 & 12.4 & 17.1 & 11.9 \\
\hline Nov-99 & 20.3 & 14.0 & 23.0 & 17.6 \\
\hline
\end{tabular}

Table 5: Mean area $\left(\mathrm{cm}^{2}\right)$ of cankers treated with brown-pigmented hypovirulent (HV) versus brown-pigmented virulent $(\mathrm{V})$ inoculum.

Measurement Date (Month-Year)

\begin{tabular}{|c|c|c|c|c|c|c|c|}
\hline & Jul-98 & Aug-98 & Sep-98 & Oct-98 & May-99 & Jun-99 & Nov-99 \\
\hline $\begin{array}{c}\text { Mean area of HV-inoculated } \\
\text { cankers }\end{array}$ & 22.1 & 70.0 & 156.1 & 269.4 & 580.0 & 711.0 & $925.6^{*}$ \\
\hline $\begin{array}{c}\text { Mean area of V-inoculated } \\
\text { cankers }\end{array}$ & 31.2 & 83.3 & 176.6 & 269.4 & 580.8 & 660.2 & $1294.0 *$ \\
\hline
\end{tabular}

* - Significant difference between area of OHV- from BRV-inoculated cankers 


\section{Bark Plugs Obtained from Cankers}

Cryphonectria parasitica cankers were sampled on ten dates to monitor the recovery of hypovirulent isolates over time and compare the recovery of hypovirulent isolates from portions of cankers established before versus after hypovirus challenges. From November 1998 thru February 2000 a total of 3,618 bark samples were obtained from 24 test cankers, of which 2,176 yielded orange-pigmented virulent isolates (60.1\%), 813 orange-pigmented hypovirulent isolates ( $22.5 \%$ ), and 629 contaminated samples (17.4\%). From 16 cankers used as controls, 1,191 orange-pigmented virulent samples (63.3\%), 684 contaminated cultures (35.8\%), and 17 samples scored as hypovirulent $(0.9 \%)$ were obtained from 1,892 bark plug cultures. A total of 4 brown-pigmented isolates were recovered in portions of cankers established after hypovirus challenge but they were classified as contaminants for statistical comparisons due to their rare occurrence. The isolate cultured from each sample point is given in Appendix C.

Because of the limited number of suitably-sized trees, there were insufficient numbers of control cankers for parametric t-test comparisons between means of isolates recovered from control versus test cankers. However, on June 25, 1999 (Sample 8) control cankers from both Experiment II and III cankers were sampled, giving a large enough data set for comparisons. Significantly more orange-pigmented hypovirulent samples were recovered from test cankers than control cankers ( $\mathrm{F}=30.30, \mathrm{P}<0.0001)$. Because these comparisons involve isolations taken from cankers outside this data set, further results are given in Chapter III.

All inoculations to establish cankers were successful in inciting infections. Further, there was $100 \%$ transmission of hypovirus to all cankers challenged with hypovirulent inoculum because at least one bark plug per canker yielded an orangepigmented hypovirulent isolate over the course of sampling.

There was a significant $\left(\mathrm{R}^{2}=0.5032, \mathrm{P}=0.0216\right)$ linear response in the percent of isolates scored as orange-pigmented hypovirulent (OHV) over time (Formula 1). Given a similar sampling scheme, the linear model predicts:

$\%$ Samples OHV $=10.00237+1.76271 *$ \# Months since challenge. 
A general 1:1 linear correlation between the percentage of OHV isolates and time was found. Although there also was a significant fit using the quadratic model $\left(\mathrm{R}^{2}=0.5879\right.$, $\mathrm{P}=0.049$ ) the increase in $\mathrm{R}^{2}$ from linear to higher order polynomials was not statistically significant and the cubic model was not significant. Insufficient data were collected to determine that the data better fit a non-linear model (e.g. Logistic or Gompertz models) than the linear model. There was an increase in the recovery of hypovirulent isolates even when the cankers were "dormant" for the winter and not visibly expanding.

As previously described, not all traced canker rings were sampled in December 1998, January 1999 and May 1999 (sample periods 2, 3 and 7, respectively) as the inner rings areas were too small to yield sufficient phloem for sampling. The effects of the decreased sample numbers can be seen in Figure 8 with the smaller sample numbers reflected in smaller total bar height at those three samples.

Cankers stopped expansion for the 1998-growing season sometime in October, near the date they were challenged. Visible expansion in the following year was reinitiated in May, allowing for cankers to be measured and outlined again, then sampled. Therefore, the increase in total number of samples obtained subsequent to May 1999 was a result of larger numbers of samples taken per canker as they expanded (Figure 12). The increase in recovery of hypovirulent and contaminated isolates was very pronounced between the 1998 and 1999 growing seasons. Significantly more orange-pigmented hypovirulent $(\mathrm{OHV})$ isolates $(\mathrm{F}=17.00, \mathrm{P}=0.0002)$ and contaminated $(\mathrm{C})$ isolates $(\mathrm{F}=5.16$, $\mathrm{P}=0.0279$ ) were recovered per canker between May 1999-February 2000 than between November 1998-April 1999 (Figure 12). Conversely, significantly more orangepigmented virulent $(\mathrm{OV})$ isolates $(\mathrm{F}=35.01, \mathrm{P}<0.0001)$ were recovered per canker from November 1998-April 1999 than from May 1999-February 2000 (Figure 9). 
Table 6: Mean number of OHV, $\mathrm{OV}$ and $\mathrm{C}$ isolates recovered per canker from Samples 1 to 6 (Nov-98 to Apr-99) versus Samples 7 to 10 (May-99 to Feb-00).

\begin{tabular}{|c|c|c|c|}
\hline & OHV & OV & C \\
\hline Nov-98 to Apr-99 & 9.750 & 58.667 & 8.750 \\
\hline May-99 to Feb-00 & 24.125 & 32.000 & 17.417 \\
\hline
\end{tabular}

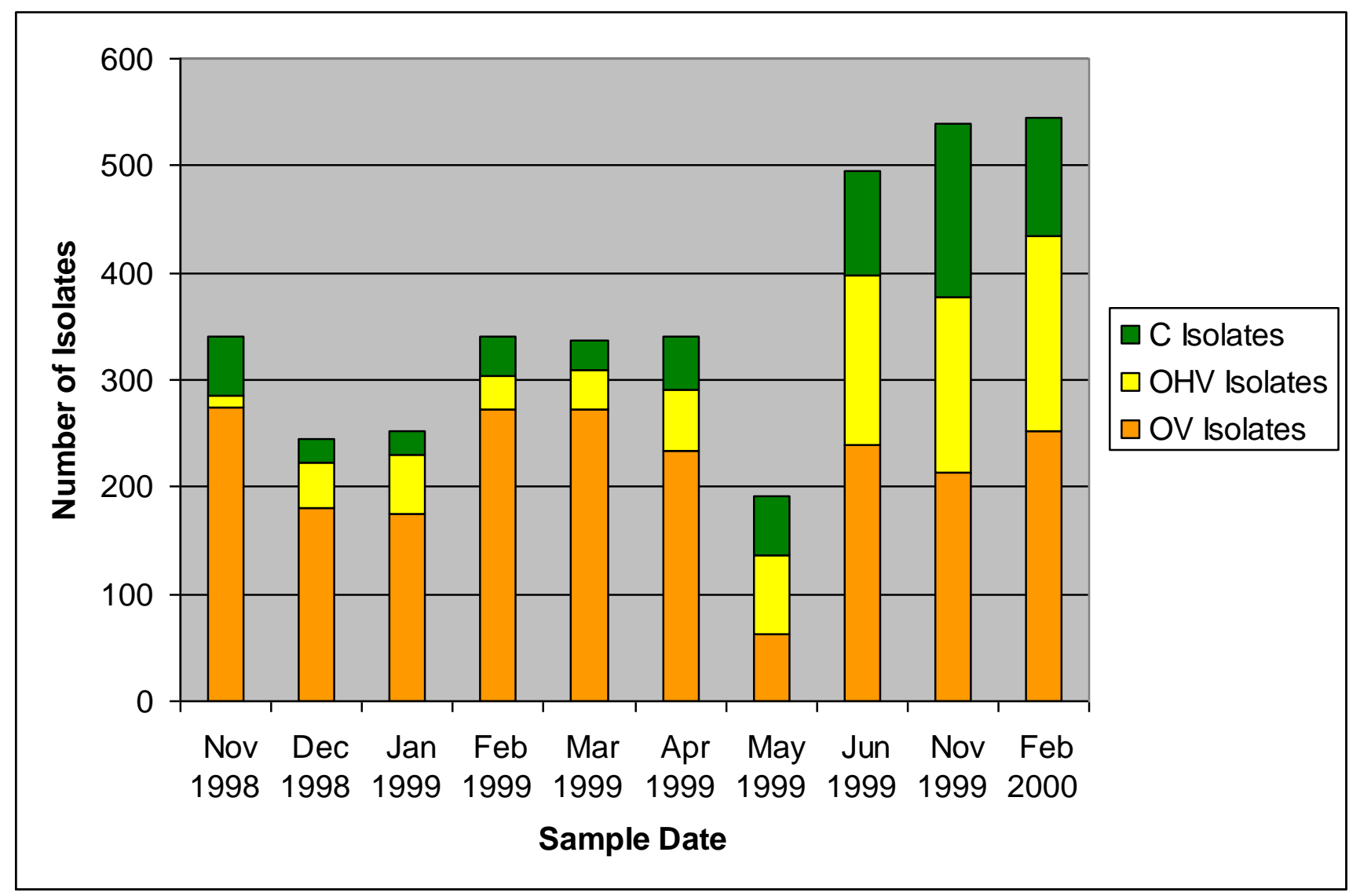

Figure 12: Recovery of orange-pigmented hypovirulent (OHV), orangepigmented virulent $(\mathrm{OV})$ or contaminant $(\mathrm{C})$ isolates from cankers over all ten sample dates. 
The distinction between isolations taken from portions of cankers established before versus those established after hypovirulent inoculum challenges could not be made until cankers had expanded past the challenge points. This did not occur until May 25, 1999 (Sample 7). Mean isolate recoveries were compared using a t-test as described earlier. No significant differences between the average number of recoveries of orangepigmented hypovirulent isolates from canker portions established before versus after hypovirus challenge were seen in May 1999 ( $\mathrm{F}=0.01, \mathrm{P}=0.9216)$, June 1999 ( $\mathrm{F}=1.79$, $\mathrm{P}=0.1876$ ), November 1999 ( $\mathrm{F}=0.88, \mathrm{P}=0.3530$ ), or February 2000 sampling dates $(\mathrm{F}=0.01, \mathrm{P}=0.9198)$. Between May 1999 and February 2000 the number of isolates scored OHV recovered from canker areas that developed after challenge increased from 38 to 90 while the number of $\mathrm{OHV}$ isolates recovered from canker areas established prior to challenges increased from 37 to 92, respectively. Recovery of orange-pigmented virulent isolates was always greater from mycelium established prior to hypovirulent inoculum challenges versus those isolations made from mycelium established after challenge (Figure 13). Although no significant differences were detected, a greater percent of hypovirulent isolates were recovered from mycelium established after versus before hypovirulent-inoculum challenge (Figure 13).

In general there was an increase in recovery of OHV in all of the outlined rings (Table 7) but there was a sharp drop-off in hypovirulent isolates in February 1999 (Sample 4) relative to the previous month. However, this trend was not repeated in February 2000 (Sample 10). Hypovirulent isolates were recovered in the greatest frequencies in the outlined rings traced after hypovirulent inoculum challenge. A decrease in the recovery of OHV isolates can be seen progressing in towards cankers centers from ring 4 and outwards toward the final extent of canker expansion in rings five to seven (Figure 14). Throughout the course of the study, the percent of samples scored OHV decreased moving in towards canker centers and outward to traced canker ring seven. 


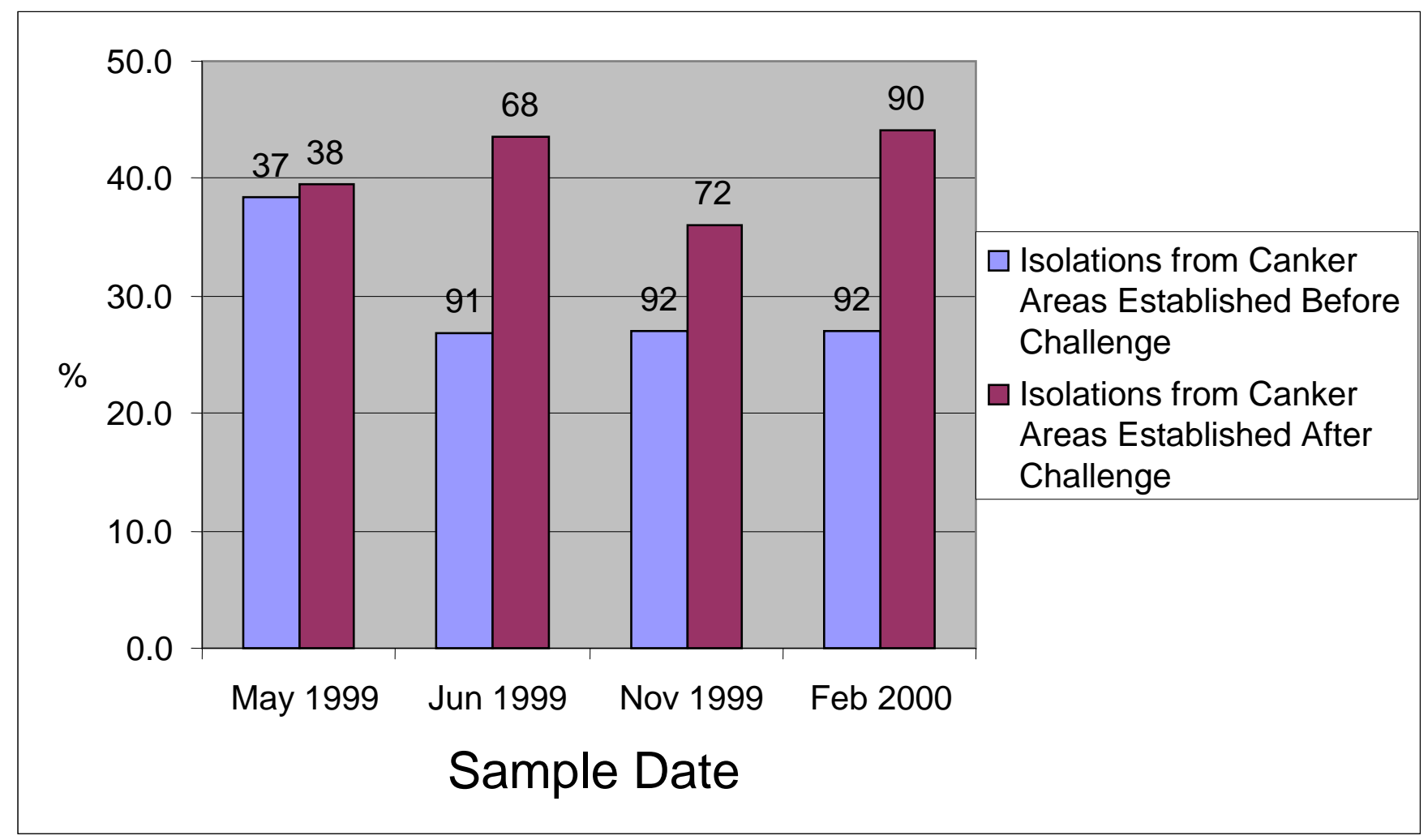

Figure 13: Percent of orange-pigmented hypovirulent (OHV) isolates from May 1999 thru February 2000 sample dates from portions of cankers established before versus after hypovirus challenge (number on tops of bars gives the number of samples each bar represents). 
Table 7: Percent of samples scored orange-pigmented hypovirulent (OHV) from outlined monthly canker expansion rings from all sample dates.

Sample
Date
\begin{tabular}{|c|c|c|c|c|c|c|c|c|c|c|}
\hline Ring $^{1}$ & Nov-98 & Dec-98 & Jan-99 & Feb-99 & Mar-99 & Apr-99 & May-99 & Jun-99 & Nov-99 & Feb-00 \\
\hline $\mathbf{1}$ & 0.0 & $\mathrm{NS}^{3}$ & $\mathrm{NS}^{3}$ & 3.6 & 0.0 & 0.0 & $\mathrm{NS}^{3}$ & 5.4 & 12.5 & 10.7 \\
\hline $\mathbf{2}$ & 0.0 & 3.1 & 9.4 & 2.4 & 3.6 & 9.5 & $\mathrm{NS}^{3}$ & 17.9 & 17.9 & 11.9 \\
\hline $\mathbf{3}$ & 4.2 & 9.4 & 16.7 & 9.4 & 13.5 & 16.7 & $\mathrm{NS}^{3}$ & 26.0 & 30.2 & 34.4 \\
\hline $\mathbf{4}^{\mathbf{2}}$ & 4.2 & 10.4 & 28.1 & 12.5 & 14.6 & 16.7 & 28.1 & 25.0 & 34.4 & 38.5 \\
\hline $\mathbf{5}$ & & & & & & & 42.0 & 52.1 & 41.7 & 41.7 \\
\hline $\mathbf{6}$ & & & & & & & & 28.6 & 35.7 & 46.4 \\
\hline $\mathbf{7}$ & & & & & & & & 16.0 & 0.0 & 40 \\
\hline
\end{tabular}

${ }^{1}$ Rings 1-to-4 were established before and rings 5-to-7 established after hypovirus challenge.

${ }^{2}$ Outlined ring that received hypovirulent inoculum challenge.

${ }^{3} \mathrm{NS}=$ Not Sampled.

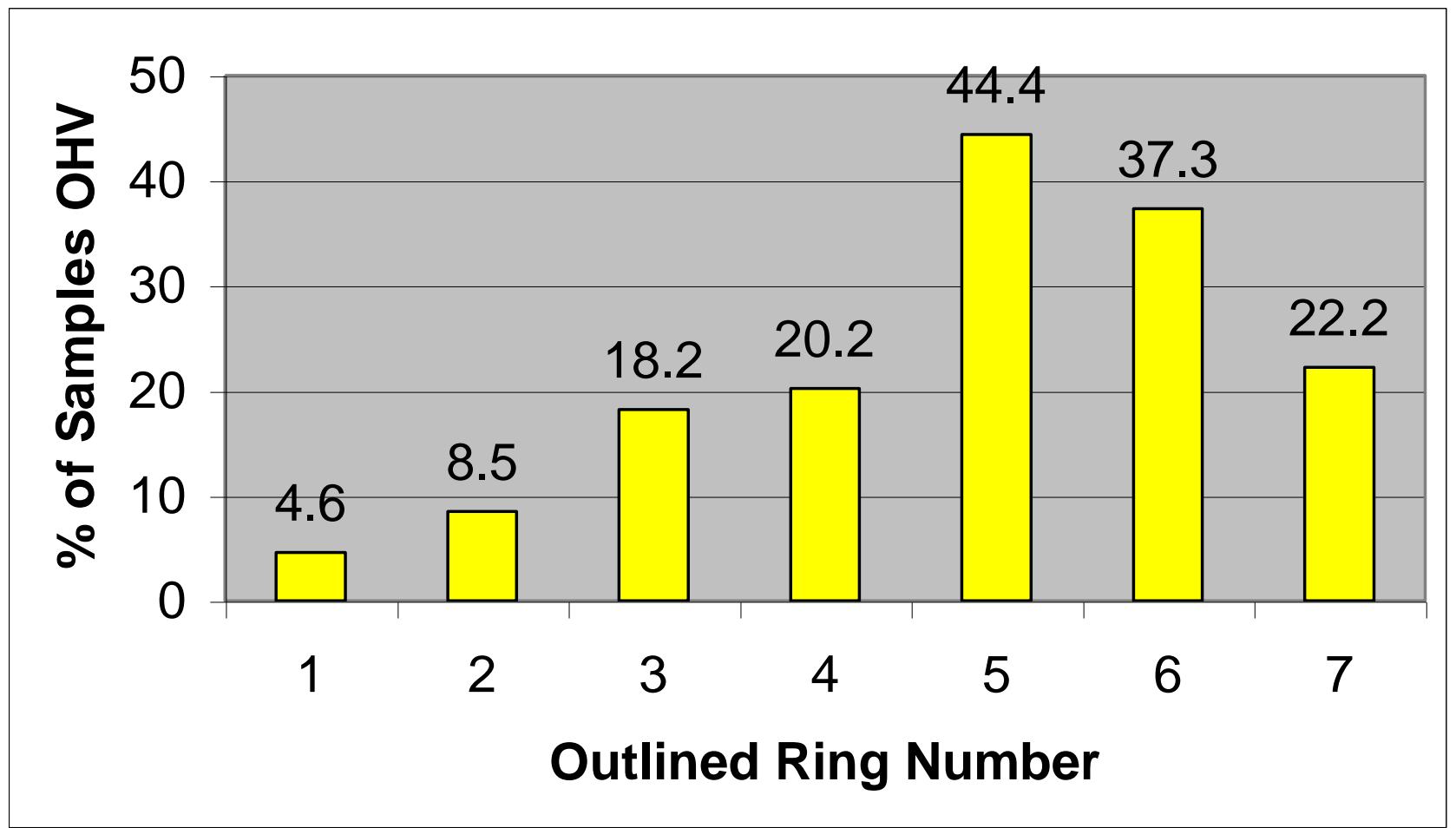

Figure 14: Percent of orange-pigmented hypovirulent (OHV) isolates per outlined canker ring recovered from cankers throughout the course of the study. 


\section{CHAPTER III-ACQUISITION OF HYPOVIRUS IN VIVO METHODS AND MATERIALS}

The primary objective of this portion of the study was to determine if fungal mycelia invading cambial, inner, and outer phloem tissues become equally colonized with hypovirus. Experiment III-A involved sampling cankers with a bone marrow biopsy tool in conjunction with those cankers from Experiment II. Experiment III-B consisted of more detailed sampling by the dissection of bark plugs into different bark layers for culturing of $C$. parasitica from outer bark, inner bark, and cambium-xylem tissues separately. Lastly, Experiment III-C, served as an additional control from sampling cankers that had been initiated with mycelium and agar plugs of each of the virulent and hypovirulent strains utilized in this study but never faced an inoculum challenge.

\section{Experiment III-A}

The purpose of Experiment III-A was to sample cankers via bark plugs to establish a baseline level of hypovirus within cankers that were treated identically to those described for Experiment II. To achieve the objective, 56 YB-2 cankers were initiated on June 25, 1998; 4 cankers per tree on 14 trees as described. Also in conjunction, cankers were monitored monthly and records of canker expansion were maintained by tracing canker margins directly onto the stems using a permanent marker on the same dates: 7/31/98, 8/24/98, 9/16/98, 10/16/98, 5/25/99, 6/25/99, and 10/29/99. At the time of tracing, canker length and width $(\mathrm{cm})$ was measured.

After allowing four months to expand, cankers were challenged on November 29, 1998 with a hypovirulent strain of $C$. parasitica to introduce hypovirus. Wounds for challenges were made using a leather punch and hammer and centered on the outer margin of the base of each canker such that half the wound was made to the cankers and half to non-colonized bark. Twenty cankers were challenged with mycelial plugs of the brown-pigmented strain 80-2C infected with Hypovirus Cryphonectria hypovirus 1-80-2. As a control, 8 cankers on two trees were challenged with mycelial plugs of the brownpigmented virulent strain 80-2C. One June 25, 1999 bark plugs were obtained using 2- 
$\mathrm{mm}$ bone marrow biopsy tools. The mean number of $\mathrm{OHV}, \mathrm{OV}$ and $\mathrm{C}$ isolates recovered per canker from $\mathrm{HV}$-inoculum $(\mathrm{n}=56)$ versus $\mathrm{V}$-inoculum $(\mathrm{n}=16)$ challenged cankers were compared using Tukey's Studentized Range (HSD) t-Test within the SAS ${ }^{\circledR}$ statistical software package (SAS Institute Inc., Cary, NC).

\section{Experiment III-B}

The purpose of Experiment III-B was to destructively sample cankers to obtain mycelium from different host tissues. Because cankers could only be sampled once using this method, each canker was also sampled using a bone marrow biopsy tool (Experiment III-A) to establish the baseline level of hypovirus colonization within cankers. To meet the objective of Experiment III-B, cankers were harvested with a chainsaw on two dates. The first harvest occurred on July 26, 1999 when 12 cankers from 3 trees were harvested. The second was on November 29, 1999 when 16 cankers from 4 trees were harvested. There were 4 fewer cankers on the first sample date because one of the trees had died and nearly the entire surface of the bark was overrun with Cryphonectria and competing fungi. Cankers were brought back to the laboratory as small 30-to-40-cm-long bolts. The cut ends of the stems were sealed with paraffin wax and placed in storage at $-20^{\circ} \mathrm{C}$ until the bark could be sampled.

On January 13, 2000, bolts were removed from storage and soaked for 30 minutes in a $10 \%$ bleach solution to surface sterilize and thaw cankers. After soaking, cankers were sampled using a sterile 2-mm bone marrow biopsy tool to remove 4 bark plugs per traced growth region from each canker. This resulted in a variable number of bark plugs per canker, as each canker had variable growth rates. After sampling, bark plugs position

was maintained by placing them in a 96-well microtiter dish. Plugs were kept in place by covering the wells with tape. Microtiter dishes were then stored at $-24^{\circ} \mathrm{C}$ until bark plugs could be cultured for Cryphonectria.

The locations of sample spots was correspond to how cankers were sampled using a bone marrow biopsy tool (top, bottom, left and right from all traced rings). However, the sampling procedure had to be modified because mycelial fans were not visible in regions of cankers that were established after hypovirus challenge and were only apparent 
in virulent, wild type and cankers treated with virulent inoculum. Therefore, the sampling procedure was modified to sampling of whole tissue types (outer bark, inner bark and cambium-xylem) of bark plugs to allow for consistent sampling of cankers in regions that were established before and after hypovirulent inoculum challenges.

Culturing of Cryphonectria from the bark plugs occurred on February 1, 2000, February 7, 2000, February 17, 2000, February 18, 2000, and April 7, 2000. Bark plugs were surface sterilized in a bleach solution for 13 minutes and rinsed in sterile water, as described previously. Following sterilization, bark plugs were divided under a dissecting microscope to dead outer bark, living inner bark, and cambial and xylem tissues. The three sections from each bark plug were cultured on GYE at room temperature under continuous bright, fluorescent lighting (Appendix A). After 3 to 4 days, Cryphonectria cultures were transferred to PDA and maintained for one week under the same conditions (Appendix A). Resulting colonies were scored based on their morphology and pigmentation by comparing them to standards. Colonies were scored as orange- or brown-pigmented virulent, orange- or brown-pigmented hypovirulent, or contaminated. Tukey's Standardized Range Test was used to compare the proportion of OHV, OV, and $\mathrm{C}$ isolates recovered from inner bark, outer bark and cambium-xylem.

\section{Experiment III-C}

The objective of Experiment III-C was to serve as an additional control by inciting cankers with each of the virulent and hypovirulent strains listed in Table 1. The

primary difference between these cankers and those described in Experiment II was that Experiment III-C cankers never faced an inoculum challenge. To meet the objective, on June 25, 199852 cankers were initiated with mycelium and agar plugs on 10 trees. Canker initiation arrangement was similar to that described for Experiment II but 6 cankers per tree were initiated with hypovirulent inoculum while 4 cankers per tree were initiated with virulent inoculum. Canker initiations were made as follows: 2 replicate trees of 4 YB-2 cankers; 2 replicate trees of 80-2C cankers; 3 replicate trees with both 3 cankers of YB-2 [CHV1 80-2] and 3 cankers of 80-2C [CHV1 80-2]; and, 3 replicate trees with both 3 cankers of YB-2 [CHV3 CoLi 11-1] and 3 cankers of 80-2C [CHV3 
CoLi 11-1]. Cankers were monitored for growth, but small size prevented hypovirulentinoculum-initiated cankers margins from being traced or measured parallel with Experiment II. Cankers incited with virulent inoculum developed typical cankers and girdled the small stems prior to the conclusion of the study, which also prevented accurate tracing and measuring of cankers.

Cankers initiated with hypovirulent mycelium were sampled using a bone marrow biopsy tool on March 26, 1999. Samples were made in a tight circular arrangement around the canker initiation point, 4 bark plugs per canker, even if the bark appeared healthy. On February 25, 2000 all cankers were sampled, 4 bark plugs per canker, with a bone marrow biopsy tool. 


\section{RESULTS}

\section{Experiment III-A}

The purpose of this experiment was to establish a baseline level of hypovirus colonization within cankers to be later sampled as part of Experiment III-B and determine if the repeated sampling of cankers over time (Experiment II) was resulting in greater recovery of contaminants. In total, 1,048 bark plugs were obtained from 56 cankers that had been challenged with hypovirulent (HV) inoculum in the previous fall. Culturing of bark plugs recovered from cankers challenged with HV-inoculum yielded: 515 (49.1\%) orange-pigmented virulent (OV), 396 (37.8) orange-pigmented hypovirulent (OHV) and $137(13.1 \%)$ contaminated isolates (C). Also, 336 bark plugs were recovered from 16 cankers challenged with a brown-pigmented virulent (V) inoculum as a control.

Culturing of bark plugs recovered from control cankers yielded: no hypovirulent isolates, 269 orange-pigmented virulent isolates $(80.1 \%)$ and 67 contaminate $(19.9 \%)$.

Significantly more OHV isolates were recovered per canker from cankers treated with HV-inoculum than controls $(\mathrm{F}=30.30, \mathrm{P}<0.0001)$. Conversely, significantly greater $\mathrm{OV}$ isolates $(\mathrm{F}=27.56, \mathrm{P}<0.001)$ and $\mathrm{C}$ isolates $(\mathrm{F}=4.50, \mathrm{P}=0.0374)$ were recovered from control cankers versus those treated with HV inoculum.

Using Tukey's t-test, the mean number of $\mathrm{OHV}, \mathrm{OV}$ and $\mathrm{C}$ isolates recovered per canker was compared to those cankers sampled on the same date as part of Experiment II $(n=24)$. No significant differences were found between the proportion of OHV isolates $(\mathrm{F}=0.14, \mathrm{P}=0.7053)$ or $\mathrm{OV}$ isolates $(\mathrm{F}=0.45, \mathrm{P}=0.5039)$. There was however, significantly more $\mathrm{C}$ isolates per canker $(\mathrm{F}=5.72, \mathrm{P}=0.0192)$ in Experiment II versus cankers sampled just one time here in Experiment III-A. Almost no difference was found between the percent of isolates scored OV from cankers sampled once versus those repeatedly sampled (Figure 15). 


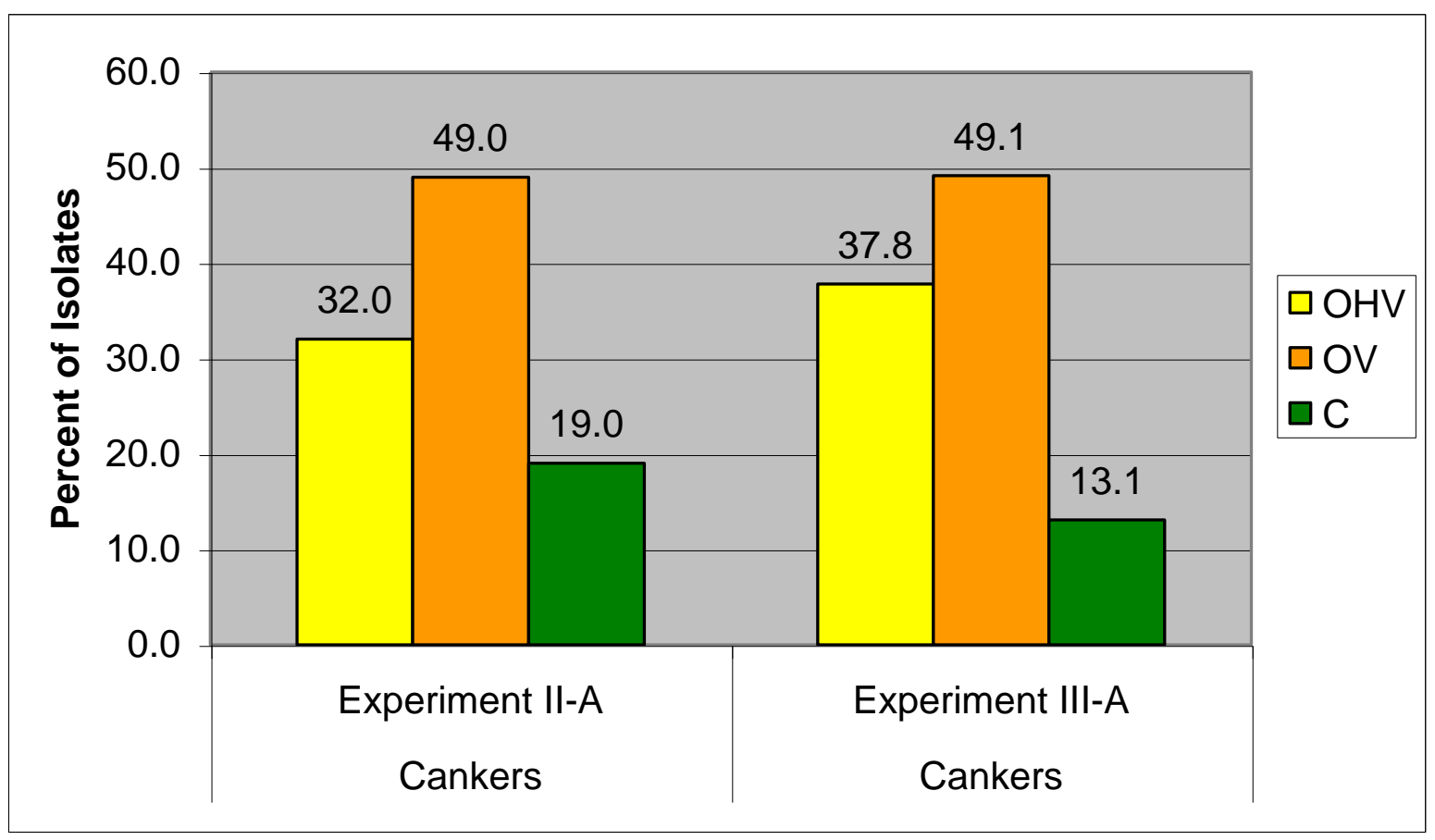

Figure 15: Frequency of orange-pigmented hypovirulent (OHV), orangepigmented virulent $(\mathrm{OV})$, and contaminated $(\mathrm{C})$ isolates recovered from Experiment II-A versus Experiment III-A cankers. 


\section{Experiment III-B}

The number of $\mathrm{OHV}, \mathrm{OV}$, and $\mathrm{C}$ isolates obtained from each tissue type was nearly identical (Table 8). No significant differences were found among any of the three host tissues. Although the proportion of OHV (Figure 16), OV (Figure 17) and C (not shown) isolates recovered per canker was equal, in a given bark plug there may have been a difference in the virulence phenotype (OV or OHV) of mycelium in different host tissue layers. In total, 344 bark plugs were obtained. Sixty-eight bark plugs (19.8\%) yielded at least one orange-pigmented hypovirulent and one orange-pigmented virulent isolate. Fifty bark plugs (14.5\%) yielded only OHV or some combination of OHV and C isolates. The remaining 226 bark plugs (65.7\%) yielded only OV or some combination of $\mathrm{OV}$ and $\mathrm{C}$ isolates.

There was a slight decrease in recovery of orange-pigmented hypovirulent isolates progressing outwards from the cambium to the outer bark (Figure 16), but no significant difference was found ( $\mathrm{F}=0.18, \mathrm{P}=0.8372)$. No significant differences were detected between $\mathrm{OV}$ isolates $(\mathrm{F}=0.23, \mathrm{P}=0.7973)$ or $\mathrm{C}$ isolates $(\mathrm{F}=0.44, \mathrm{P}=0.6454)$. Although differences were not significant, more orange-pigmented virulent and less contaminated cultures were recovered from samples taken from the inner bark.

Table 8: Recovery of orange-pigmented virulent (OHV), orange-pigmented virulent $(\mathrm{OV})$, and contaminated $(\mathrm{C})$ isolates from outer bark, inner bark, and cambiumxylem tissues from 344 dissected bark plugs.

\begin{tabular}{|c|c|c|c|c|}
\hline & & $\begin{array}{c}\text { Host } \\
\frac{\text { Tissue- }}{\text { Type }} \\
\text { Sampled }\end{array}$ & & \\
\hline Isolate Recovered & $\frac{\underline{\text { Outer }}}{\text { Bark }}$ & $\frac{\underline{\text { Inner }}}{\text { Bark }}$ & $\frac{\text { Cambium- }}{\underline{\text { Xylem }}}$ & $\underline{\text { Sum }}$ \\
\hline OV & 200 & $\mathbf{2 0 7}$ & $\mathbf{1 8 3}$ & $\mathbf{5 9 0}$ \\
\hline OHV & 61 & 68 & 75 & 204 \\
\hline C & 81 & 67 & 85 & 233 \\
\hline Sum & 342 & 342 & 343 & 1027 \\
\hline
\end{tabular}




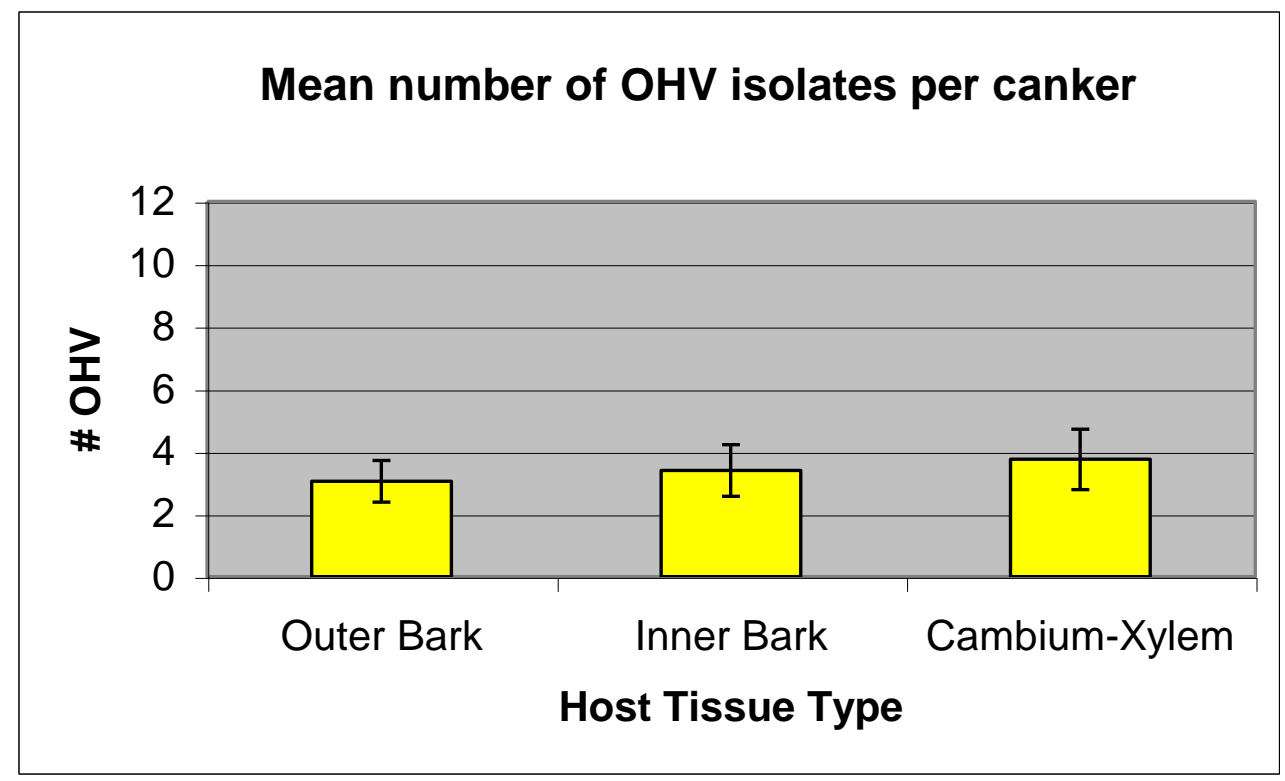

Figure 16: Mean number and standard error of orange-pigmented hypovirulent (OHV) isolates recovered from inner bark, outer bark and cambium-xylem.

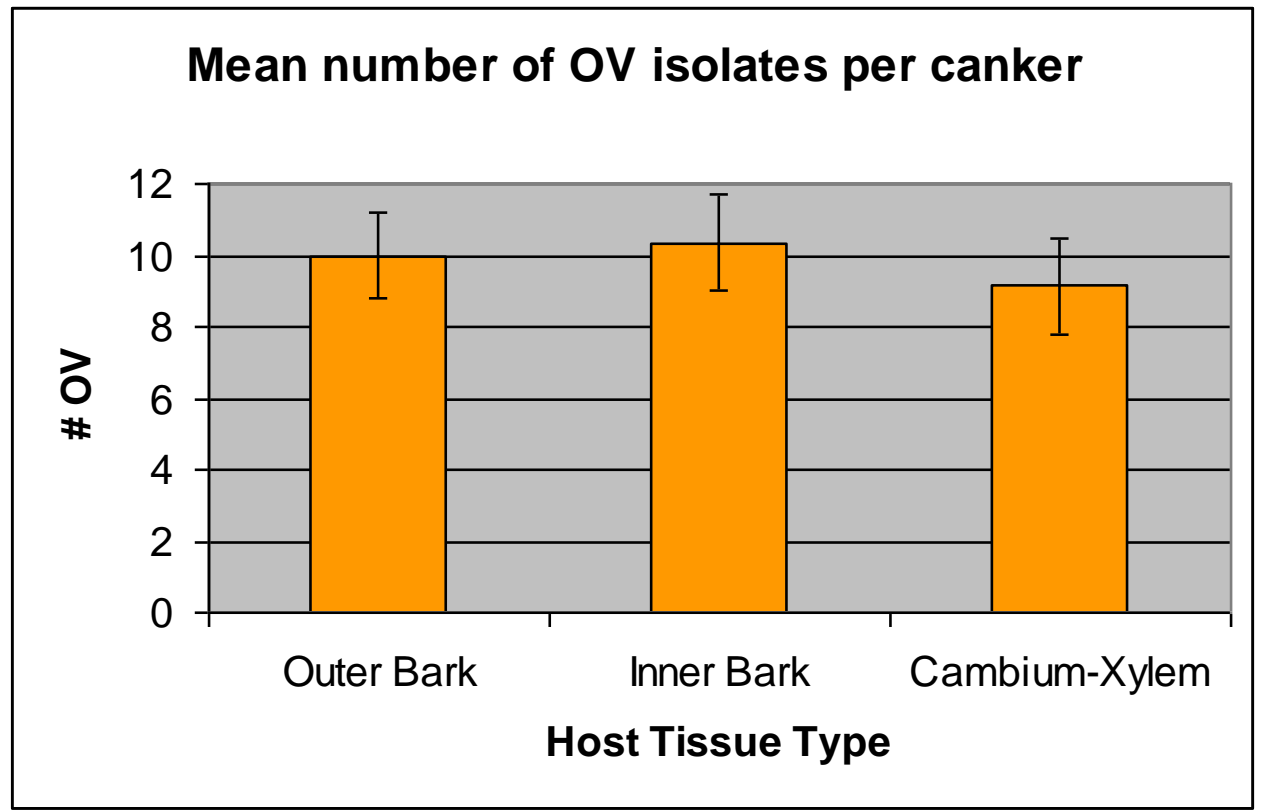

Figure 17: Mean number and standard error of orange-pigmented virulent (OV) isolates recovered from inner bark, outer bark and cambium-xylem host tissues. 


\section{Experiment III-C}

The objective of Experiment III-C was to determine the stability in vivo of morphology and pigmentation phenotype for all brown- and orange-pigmented and virulent or hypovirulent isolates used as part of this study. Cankers using each of the isolates were wound-initiated and then sampled once (virulent isolate-initiated cankers) or twice (hypovirulent isolate-initiated cankers). Sampling of HV-inoculum-initiated cankers occurred on March 26, 1999 and of all cankers on February 25, 2000. Cankers were monitored monthly along with those discussed in Chapter II but were not traced because hypovirulent-inoculum initiated cankers never visibly expanded. From all of the cankers except those initiated with brown-pigmented 80-2C [CHV3 CoLi 11-1], where contamination was greater, the primary isolates recovered on both sampling dates matched those used to initiate the cankers (Table 9). "Other C. parasitica isolate" recovered meant an isolate that did not match morphology or pigmentation of the cankerinciting isolate; these isolates typically were orange-pigmented virulent isolates, i.e. "wild types" (Table 9). No statistical comparisons were conducted as this experiment was only intended to be informational. 
Table 9: Isolates recovered from unchallenged cankers (Experiment III-C) on the dates of April 26, 1999 and February 25, 2000.

\begin{tabular}{|c|c|c|c|c|c|c|}
\hline & $\begin{array}{c}\text { 4/26/1999 } \\
\text { Sample } \\
\underline{\text { Date }}\end{array}$ & $\begin{array}{c}\text { Isolate } \\
\text { Recovered }\end{array}$ & & $\begin{array}{c}2 / 25 / 00 \\
\text { Sample } \\
\text { Date }\end{array}$ & $\begin{array}{c}\text { Isolate } \\
\text { Recovered }\end{array}$ & \\
\hline$\frac{\text { Inoculum Isolate }}{\text { [Hypovirus] }^{\star}}$ & $\begin{array}{c}\frac{\text { Inoculum }}{\text { Isolate }} \\
\text { Recovered }\end{array}$ & $\frac{\text { Contaminant }}{\text { Recovered }}$ & $\frac{\text { Other } C .}{\frac{\text { parasitica }}{\text { Isolate }}}$ & $\frac{\text { Inoculum }}{\text { Isolate }}$ & $\frac{\text { Contaminant }}{\text { Recovered }}$ & $\begin{array}{c}\text { Other } C . \\
\frac{\text { parasitica }}{\text { Isolate }} \\
\text { Recovered }^{\star *}\end{array}$ \\
\hline MC2 [CHV1 80-2] & 26 & 10 & 0 & 23 & 3 & 10 \\
\hline MC2 [CHV3 CoLi 11-1] & 29 & 6 & 1 & 27 & 4 & 5 \\
\hline $80-2 \mathrm{C}[\mathrm{CHV} 180-2]$ & 16 & 13 & 7 & 30 & 3 & 3 \\
\hline 80-2C [CHV3 CoLi 11-1] & 10 & 22 & 4 & 29 & 2 & 5 \\
\hline MC2 & $\mathrm{NS}^{* * *}$ & $\mathrm{NS}^{* * *}$ & $\mathrm{NS}^{* * *}$ & 24 & 0 & 0 \\
\hline $80-2 C$ & $N S^{* * \star}$ & $N S^{* * \star}$ & $\mathrm{NS}^{* \star \star}$ & 17 & 1 & 6 \\
\hline
\end{tabular}

*Isolate used to initiate cankers recovered.

**Isolate of $C$. parasitica was recovered that was not the isolate used to incite canker. $* * *$ Not sampled. 


\section{DISCUSSION}

Use of hypoviruses as biological control agents to manage for the chestnut blight disease has met with limited success in North America. Reasons for this limited success have been variously attributed to a number of causes, including: a complex system of vegetative incompatibility within the fungus; lack of hypovirus infection of sexual and asexual reproductive structures; and, lack of an efficient hypovirus vector. This research

project was prompted by results from other field studies involving chestnut blight cankers in West Salem, WI, and previous work conducted at West Virginia University (Balbalian, 1998). Those studies demonstrated that recovery of $C$. parasitica isolates from cankers could vary, even when the same exact cankers and locations on cankers were being resampled. Hypoviruses have been shown to first colonize the perimeter of cankers then progress inwards towards canker centers over time (Shain and Miller, 1991) and to rapidly colonize colony margins in laboratory experiments at a rate three-to-four times greater than colony expansion (Martin and Van Alfen, 1991). In another study, "whitepigmented" hypovirulent strains of $C$. parasitica were found randomly distributed in cankers that had been treated with hypovirulent strains 15-16 years prior (Griffin, 1999). However, the mechanism by which hypoviruses become established, replicate and move through the host fungal thallus is poorly understood. Therefore, the first objective of this study was to determine if mycelium in older or younger portions of cankers is colonized by hypovirus equally; as mycelial age, an indirect measure of fungal physiology, was hypothesized to be an important variable affecting recovery of hypovirulent isolates. The second objective of this study was to determine the age at which mycelium is no longer able to acquire hypovirus.

The first step for this study was to assemble a set of virulent and hypovirulent isolates with stable phenotype in culture. Isolates selected were vegetatively-compatible with one another as vegetative incompatibility was presumed to be the primary barrier to successful transmission of hypoviruses (MacDonald and Fulbright, 1991). However, it has been shown that vegetative incompatibility does not necessarily prevent transmission (Anagnostakis and Day, 1979 and Huber, 1996). Use of the brown-pigmented hypovirulent strain $80-2 \mathrm{C}$ to deliver hypovirus to orange-pigmented virulent strain $\mathrm{YB}-2$ 
made it possible to distinguish the virulent from hypovirulent phenotype or from subsequent growth of the hypovirulent inoculum. Therefore, recovery of orangepigmented hypovirulent isolates indicated transmission of hypovirus, not growth of the strain used for challenge. Hyphae of the brown isolate $80-2 \mathrm{C}$ were not expected to cohabitate where mycelium of the orange isolate YB-2 was already established (Martin and Van Alfen, 1991). Throughout the study, brown versus orange virulent or white hypovirulent pigmentation turned out to be a very stable marker to distinguish the isolates. No brown-pigmented virulent or brown-pigmented hypovirulent isolates were ever subcultured from portions of colonies that appeared orange-pigmented. Scoring fungal background (orange-pigmented YB-2 or brown-pigmented 80-2C) and hypovirus infection based solely on morphology and pigmentation (Elliston, 1985 and MacDonald and Fulbright, 1991) appeared to be sufficient. Phenotypic stability was assumed based on maintenance of standards and PCR verification that Lynn Geletka performed (Appendix A), and recovery of all isolates from unchallenged cankers as described in Experiment C (Chapter III).

One potential problem with the experiments was that without pairing cultures to determine vegetative compatibility, there was no way to tell the difference between wildtype, orange-pigmented virulent strains of $C$. parasitica and orange strain YB-2 when OV strains were obtained from cankers. However, even under bright light which inhibits colonies from fusing, hypovirulent colonies all anastomosed indicating vegetative compatibility. Other potential problems with the methodology may have included a lack of a means to distinguish replication of hypovirus in sutu from translocation within mycelium. Additionally, I was unable to determine if hypovirus transmission occurred upon culture. The only indication of the actual level of cross-contamination within cankers came from culturing of isolates that were not used to incite the additional control cankers as described in Chapter III (Table 8). Previous microscopic examinations of hyphal anastomoses between hypovirulent and virulent hyphae indicated that passive translocation likely does not occur (Newhouse and MacDonald, 1991).

The two experiments described in Chapter I were intended to determine the age at which colonies are no longer able to acquire hypovirus (Experiment I-A), compare recovery of hypovirulent isolates from younger versus older portions of colonies 
(Experiment I-A), and determine if hypovirus can be transmitted through virulent mycelium (Experiment I-B). In Experiment I-A, mycelium and agar plugs of 80-2C [CHV1 80-2] was used to challenge colonies of YB-2 weekly for seven weeks beginning with co-inoculations of zero-week-old colonies. Subsequent sampling recovered isolates from mycelium that was established before or after the time of hypovirus challenge. Only two out of $563(0.4 \%)$ isolates obtained from mycelium established one week prior to hypovirulent inoculum challenge were scored as hypovirulent. In Experiment A, both $\mathrm{HV}$ isolates that were obtained from mycelium established prior to hypovirulent inoculum challenge came from the same colony. This may indicate contamination from the sampling technique because at least one prior [hypovirulent] sample was obtained beyond the challenge point and sterilization of the scalpel was only performed every four samples. Therefore, if no hypovirulent isolates were ever recovered from mycelium established prior to hypovirus challenge, the objective to determine the age at which older mycelium is no longer able to acquire hypovirus was not met because it never occurred.

Over six weeks of isolations, no hypovirulent strains were ever recovered from mycelium established two-to-six weeks prior to challenge. Conversely, the proportion of hypovirulent isolates increased from $46 \%$ to $94 \%$ of isolates obtained from mycelium that extended from one to three weeks after challenge, respectively. Clearly, hypovirus could be successfully transmitted to colonies, but "conversion" if it occurred was not conversion of existing, virulent mycelium rather it was "conversion" of the future colony extension that happened to be hypovirulent. Conversion appears to be a poorer word choice than simply "infection" as little evidence could be found that anything beyond conversion of a potential outcome (i.e. the hypovirulent phenotype) actually occurred. Since only colony extension subsequent to challenge yielded hypovirulent isolates, no trend was discovered that indicated an increase or decrease in the level of hypovirulence over time that could no be explained by a larger area of now hypovirulent extending mycelium. This indicated that phenotype is determined on formation of tissue and does not vary thereafter.

Experiment I-B was intended to overcome some of the limitations of Experiment A by comparing challenges made with two different hypoviruses [CHV1 80-2] and [CHV3 CoLi 11-1], to hypovirus-free isolate. Arrangements for challenges occurred as 
co-inoculations of zero-week-old colonies and challenges at or $0.5 \mathrm{~cm}$ behind colony leading edge of one-week-old colonies. With at least one recovery of a hypovirulent sample from a colony indicating successful hypovirus transmission, hypovirus was transmitted in $78.3 \%$ of challenges made as co-inoculations, $73.3 \%$ of challenges to colony leading edges, but only $11.7 \%$ of challenges located behind the colony leading edge. As in Experiment A, challenges to the colony leading edge with either hypovirus resulted in less than $1 \%$ recovery of hypovirulent isolates from mycelium established prior to challenge. When challenges were made behind the colony leading edge, no significant differences were found among recovery of hypovirulent isolates from controls. The slight recovery of hypovirulent isolates from challenges behind the colony margin may have resulted from hyphal response or growth from severed mycelium by the scalpel used to place the plugs for challenge or possibly even new hyphal growth to utilize the nutrients at the bottom of the agar plugs. Overall, these findings supported the concept that hypoviruses can spread rapidly in the tangential direction at colony leading edges and that radial movement of hypoviruses back towards infection origin is more limited (Martin and Van Alfen, 1991). However, radial movement appeared to be nonexistent rather than "limited". Further, the findings did not support the findings of 30 $\mathrm{mm}$ /day radial translocation of hypovirus because virulent mycelium "washed over" the challenge point and typically some virulent isolates could still be recovered from mycelium established after challenge (Figure 5). The appearance of the mycelium gave a good estimate of the isolates that were later recovered from that sector; sectors that appeared virulent typically were and the same held for hypovirulent isolates. All brownpigmented hypovirulent isolates were recovered from brown-pigmented mycelial fans that expanded after challenge.

The purposes of the first field experiments were to determine if mycelium in older or younger portions of cankers support hypovirus colonization by characterizing the spread of [CHV1 80-2] hypovirus over time. In Experiment II-A, isolations were made on ten dates while in the otherwise identical Experiment II-B, isolates were obtained one time, on June 25, 1999. In Experiment II-A, hypovirulent isolates were recovered in at least one instance from all cankers challenged with mycelium and agar plugs of 80-2C [CHV1 80-2]. This overall level of "canker conversion" was consistent with a prior 
study where hypovirulent strains were obtained from $83.3 \%$ to $100 \%$ of bark samples (Shain and Miller, 1992). Shain and Miller, 1992 represents the only report about hypovirus recovery based on re-sampling the exact same fungal structures within cankers (e.g., conidia producing cirrhi). They describe that even one year after transmission of hypovirus to the underlying fungal thallus, cirrhi continue to produce virulent conidia. Unfortunately, field experiments A and B described in Chapter II were limited to reisolations in the same general areas as opposed to necessarily re-sampling the same thallus over time. Therefore, re-isolations made in the same area were potentially recovering wild-type $C$. parasitica or even reactionary growth by isolate YB-2 in newly created wounds in the bark.

Based on the study by Shain and Miller, 1992, full colonization of canker margins was expected within three weeks after challenge. However, sampling of the traced ring, where hypovirus challenge occurred, yielded a range from $4.2 \%$ to $38.5 \%$ of isolates scored hypovirulent from one month to one year following challenge (Table 7). As expected, the innermost, oldest portions of cankers yielded the fewest hypovirulent isolates. Over the full course of Experiment II-A, sampling of the first traced ring resulted in $0.0 \%$ to $12.5 \%$ of isolates scored hypovirulent while $0.0 \%$ to $17.9 \%$ of the isolates recovered from the second traced growth ring were scored hypovirulent. The low percentage of hypovirulent isolates recovered from these cankers was more similar to poor results from canker challenges using bark patches at the end of the growing season (Balbalian, 1998) than to the observations made by Shain and Miller, 1992 where more than $80 \%$ of isolates recovered were scored hypovirulent. These findings seem inconsistent with the hypothesis that challenging cankers at the end of the growing season may not be as reliable as challenges made to actively growing cankers (Balbalian, 1998) because hypovirus was successfully transmitted to cankers, but hypovirus establishment within cankers was never in the $80-100 \%$ range as reported by Shain and Miller, 1992 for hypovirulent inoculum challenges made during the early summer.

Brown-pigmented hypovirulent isolates were recovered very infrequently from cankers. The only locations from which they were recovered came from portions of cankers established after challenges, located to the outside of the hypovirulent-inoculum challenge point. Therefore, no hyper-colonization of cankers by the brown-pigmented 
strain was detected and recovery of a hypovirulent isolate indicated successful transmission of hypovirus not ramification of the brown fungal thallus.

From cankers, the greatest numbers of hypovirulent isolates were recovered from portions of cankers established after challenges with hypovirus, but no significant differences could be detected between recoveries of isolates from portions of cankers established before versus after challenge. The greatest recovery of hypovirulent isolates occurred in the traced canker ring immediately outside of the ring of challenge (typically the fifth traced area) where a range of 41.7 to $52.1 \%$ of the isolates was scored hypovirulent. As cankers continued to expand past the fifth traced ring, the level of hypovirulence decreased. While sampling of the fifth and sixth traced canker ring, which was only possible in the spring and winter following challenge, typically yielded twice as many hypovirulent isolates as samples from the ring of challenge, the outermost, seventh traced ring yielded only 0.0 to $40.0 \%$ hypovirulent isolates. One possible explanation for this is only mostly hypovirus-free, virulent cankers had expanded enough to have a seventh traced canker ring as only five of 24 cankers had expanded sufficiently by the last sample to have the ring traced. Low number of replications is the simplest explanation for why no significant differences were detected between portions of cankers established before versus after challenge.

The purpose of Experiment II-B was to determine if the repeated sampling of cankers as part of Experiment II-A was resulting in a lower recovery of hypovirulent isolates that might otherwise be found. This lower recovery of hypovirulent isolates may have occurred because of increased recovery of contaminated cultures from cankers sampled repeatedly. Between isolates recovered from cankers sampled on June 25, 1999, significantly more contaminated isolates $(\mathrm{P}=0.0192)$ were recovered per canker from Experiment II-A than II-B. No statistically significant differences between the mean numbers of orange-pigmented virulent or orange-pigmented hypovirulent isolates recovered per canker were found. However, because the total number of orangepigmented virulent isolates between Experiments II-A and II-B was nearly identical (49.0\% and $49.1 \%$ of the samples scored OV, respectively) the greater amount of contaminated isolates was at the expense of recovering hypovirulent isolates. 
In Experiment II-A, following initiation in June 1998, they were allowed to expand until October 1998 before facing a hypovirulent inoculum challenge. No significant difference in canker area was observed between cankers treated with virulent versus hypovirulent inoculum until November 1999. No correlation between bark thickness and recovery of hypovirulent isolates was discovered. C. parasitica grew, and was colonized by hypovirus, equally in thin-barked trees (bark thickness 1.27 to 2.54 $\mathrm{mm}$ ) as in thick-barked trees (bark thickness $3.81 \mathrm{~mm}$ or greater). Cankers initiated with hypovirulent brown- or orange-pigmented strains as part of Experiment III-B did not expand as much as cankers initiated with virulent inoculum; they failed to expand throughout the duration of the study. However, very small cankers must have resulted from inoculations with hypovirulent mycelium because sampling from the immediate inoculation area typically yielded the isolates used to initiate cankers.

Recovery of hypovirulent isolates from bark plug sampling might be "conversion" of existing virulent mycelium to the hypovirulent phenotype as the literature seems to imply, or simply the subsequent expansion of hypovirulent mycelium as was implied in the laboratory tests conducted as part of this study. Distinguishing those two possible alternatives within cankers would be very difficult, however because it was impossible to determine if hypovirus transmission occurred upon culture. Future work might include determining the rate and mode of passive or active viral transport within the fungal thallus and repeated sampling of reproductive structures over time to determine if or how the fungus is preventing or even escaping viral infection. Within cultures, hypoviruses were not consistently shown to be able to colonize, or even move through, mycelium established prior to challenge, conflicting with prior reports (Anagnostakis and Day, 1979 and Martin and Van Alfen, 1991). Even after six weeks of growth, established, virulent mycelium remained virulent regardless of the amount of the petri dish surface overrun with hypovirulent fungus. Results from laboratory experiments were difficult to extend to the findings from fieldwork because the same mycelium from a canker could not be repeatedly sampled over time. However, it was clear that even under potentially optimum circumstances where inoculum reservoir, movement, compatibility and transmission barriers to hypovirus spread are overcome, hypovirus was not capable of ramification throughout cankers. 
The concept of "conversion" to the hypovirulent phenotype may be used accurately when referring to samples obtained from hypovirulent-appearing mycelium subsequent to challenge (Anagnostakis and Day, 1979 and this thesis) but perhaps not to cankers where "conversion" to HV has not been demonstrated. Unfortunately, conversion of pre-existing virulent mycelium to hypovirulence was never fully demonstrated in vitro or in vivo as part of this study because mycelium could not be directly sampled twice. However, results from Experiment I strongly indicated that virulent hyphal extension does not precede hypovirus colonization or else greater variation in recovery of hypovirus should have been demonstrated. One explanation for the variable recovery of hypovirulent isolates from cankers over time is that hyphal ramification within dead host cells is itself variable and only those hyphae actively growing following hypovirus challenge exhibit the hypovirulent phenotype when subcultured. Further, variability in recovery of HV isolates over time could simply be an indirect measure of fungal physiology, i.e. greater recovery of $\mathrm{HV}$ isolates only indicates greater hyphal growth at the time of challenge not increasingly or decreasingly "converted" mycelium over time. Evidence to the contrary is suggested by isolations from the outermost traced ring 7 where very few HV isolates were recovered. However, even in that case there was no way to know if thallus responsible for canker extension or hyphal colonization of dead cells following mycelial fans yielded the hypovirulent isolates (Hebard, et al., 1984). Results from sampling unchallenged cankers as part of Experiment III-C indicate a high level of Cryphonectria "contamination" also is possible. "Other" isolates were typically orange-pigmented virulent, presumably "wild-type" infections, making characterizations about the identity of the fungus. Future studies might benefit from using the brown virulent isolate to initiate infections, but for the purpose of tracing cankers for this work it was felt that the brown cankers are more difficult to trace (M. Double, pers. comm.). Some canker extension probably was due to hyper-infections by other $C$. parasitica isolates based on the recovery of the wild-type isolates from $\mathrm{HV}$-initiated cankers as part of Experiment III-C.

The few cases where pre-existing mycelium acquired hypovirus in cultures were not significantly different from the virulent control. Based upon the culture work as part of this study and sampling on conidia (Shain, 1991) one explanation for recovery of 
hypovirulent isolates from older portions of cankers is the result of continued fungal hyper-colonization of host tissues and single hyphae colonizing and ramifying throughout dead cells (i.e. mycelium established after challenge). Successful deployment of hypoviruses as biocontrol agents may continue to be hampered if cankers fully treated with hypovirus are still able to sporulate and expand from virulent portions of canker thalli.

Chapter III consisted of two unrelated experiments. The purpose of Experiment A was to characterize the location of hypoviruses within cankers by separately culturing mycelium from outer bark, inner bark and cambium-xylem tissues. After some preliminary tests, the procedure settled upon was to dissect bark plugs into outer bark, inner bark and cambium-xylem tissues using a scalpel and forceps with the aide of a dissecting microscope. Other experimental procedures attempted included cutting cankers into long strips and splitting $1 \mathrm{~cm} \times 1 \mathrm{~cm}$ bark patches into sheets. In the first attempt, $1 \mathrm{~cm}$ wide canker strips were examined under a dissecting microscope and all visible mycelial fans at the approximate center of all traced canker rings were sampled using a scalpel. This was impractical because mycelial fans were not evident past the point of hypovirulent inoculum challenges and in general mycelial fans were very difficult to locate. In the second procedure, attempts were made to split $1 \mathrm{~cm} \times 1 \mathrm{~cm}$ bark patches into tangential sheets to expose different mycelial fans. This technique was impractical because the bark did not split apart easily at the mycelial fans as was hoped, but instead was split at structural "weak spots" that varied with the bark. Both of these approaches apparently worked reasonably well with wild-type, virulent cankers, perhaps indicating that only healthy, hypovirus-free cankers produce robust mycelial fans that are easy to locate with dissection. Dissection of bark plugs obtained with a bone marrow biopsy tool was settled on because it did not require sampling of indistinct mycelial fans. Furthermore, under magnification of the dissecting microscopes outer bark, inner bark, and cambium-xylem tissues can be differentiated.

C. parasitica is known to obtain nutrients from host cells from the three tissues sampled (Keefer, 1914). Hypovirulent mycelium takes more time than virulent mycelium to overcome induced host defenses such as lignification of cell walls and wound periderm formation (Hebard, Griffin and Elkins, 1984). Therefore, the hypothesis 
that greater numbers of virulent isolates would be recovered from the cambium-xylem interface versus other bark tissues was tested. No significant difference in the numbers of orange-pigmented hypovirulent, orange-pigmented virulent or contaminated isolates per canker was discovered among cultures from outer bark, inner bark and cambium-xylem tissues. Recovering the greatest proportion of hypovirulent and contaminated isolates from the cambium-xylem tissues was an unexpected result as the living, and presumably most reactive host cells occur are in these tissues. The outermost bark yielded the lowest level of contaminated organisms compared to the other tissues; this may have been due to the bleach sterilization killing all surface contaminants leaving only organisms truly established (e.g. Trichoderma sp.) within the bark to culture.

The purpose of Experiment $\mathrm{B}$ was to serve as an additional control to determine stability within cankers of the morphological and pigmentation phenotypes used to score cultures for all of the virulent and hypovirulent isolates. Cankers initiated with 80-2C [CHV3 CoLi 11-1] failed to expand throughout the course of the study, which was an expected result as this particular CHV3-type hypovirus is very debilitating. Cankers initiated with 80-2C [CHV1 80-2] expanded very slowly and by the end of the study only very small cankers were apparent. Therefore, the cankers as part of this study were not measured or traced in conjunction with experiments described in Chapter II. Despite failure of cankers to expand, the hypovirulent isolates used to incite the cankers were recovered on both sample dates. Contaminating fungi and competitive, virulent $C$. parasitica isolates were also recovered from these cankers. In some cases, the competing fungi was the opposite pigmented hypovirulent strain. This may have been the result of bone marrow biopsy tool contaminating the sample or spread of the isolate up or down the tree stem. However, in general, the isolate used to inoculate wounds to incite a canker was the isolate predominately recovered from the canker (Table 8). From cankers initiated with hypovirulent mycelium, competing strains of C. parasitica comprised $8.3 \%$ and $16.0 \%$ of the samples on April 26, 1999 and February 25, 2000, respectively. At the same time, contaminating fungi equaled $35.4 \%$ and $8.3 \%$ of the samples, respectively. The higher level of contamination that was observed in April 1999 relative to February was probably related to environmental conditions favorable to spore dissemination. 
Cankers incited with YB-2 yielded all orange-pigmented virulent isolates. Isolations from cankers incited with $80-2 \mathrm{C}$ yielded $70.8 \%$ brown-pigmented virulent, $25 \%$ orange-pigmented virulent and $4.2 \%$ contaminated isolates. Orange-pigmented isolates recovered from cankers incited with 80-2C could have originated from YB-2 cankers from the same stem or wild-type cankers.

Results from the field experiment indicated that despite successful transmission of hypovirus to most of the cankers, recovery of hypovirulent isolates was very sporadic from different portions of cankers throughout the entire course of the study. Interestingly, despite introduction of hypovirus at one basal location on cankers, hypovirulent isolates were obtained in equal numbers from the north, east, south and west sampling positions within each traced canker area with no significant differences detectable between sampling positions. The percent of isolates scored hypovirulent showed a linear increase from each sample date except from January 1999 to February 1999. This drop in recovery of hypovirulent isolates in February also was observed in a prior study conducted in the same forest management compartment (Balbalian, 1998). Forest Management Compartment 25 is in an exposed forest-open farmland interface and subject to strong winter conditions. Although cankers appeared to stop expanding for a winter dormancy season, hypovirus replication appeared to continue as the number of hypovirulent isolates recovered increased between November 1998 and March 1999. Interior portions of cankers, whether sampled once as part of Experiment II, or ten times over the course of a year (Experiment III), yielded low ( $<40 \%$ of samples) numbers of orange-pigmented hypovirulent isolates. Sampling of portions of cankers that had expanded after hypovirulent inoculum challenges yielded greater numbers of hypovirulent isolates than samples from previously established portions of cankers. However, even sampling mycelium established after challenge yielded far fewer hypovirulent isolates than previous reports where recovery rates of hypovirulent isolates were observed at 80-100\% of isolates (Hobbins, 1985, Shain and Miller, 1992).

Throughout the study, no attempts were made to identify contaminating fungi to the species level; rather only the relative frequency of contamination was recorded. In laboratory work, contamination was almost exclusively the result of airborne spores as the only contaminants seen were colonies of Aspergillus spp. and Penicillium spp. 
Aspergillus contamination was so widespread during test experiments to refine the procedure that large $(150 \mathrm{~mm})$ petri dishes had to be abandoned entirely in favor of standard $100 \mathrm{~mm}$ petri plates for the culture work described in Chapter I. In standard petri dishes, contaminated isolates became abundant only after colonies reached 10 weeks of age or older, at which time colonies of Penicillium would begin to overrun the $C$. parasitica colonies. Usage of large numbers of replications for Experiment I meant that no colonies were sampled twice which presumably then standardized the type and species of contamination organisms because colonies were all similarly subject to the same potential contamination times.

A wide variety of contaminants, including fungi, bacteria, and Oomycetes were recovered from bark plugs. Primary contaminants included Aspergillus spp., Penicillium spp., Pestilotia spp., and Trichoderma spp., with many other organisms being recovered at lesser frequencies. Contaminating fungi were not examined in detail because necrotic cankers, let alone healthy bark, is host to a multitude of competing organisms in a continually changing dynamic (Shigo, 1967).

The final objective of this study was to characterize the nature of the "gelatinous zone" canker region by determining the virulence phenotype of mycelium within or in close proximity to the gelatinous zone and the tissue(s) that comprise the gelatinous zone. The gelatinous zone is a thin band of transparent cells located at canker margins. In the literature the gelatinous zone has been variously described as "a darker gelatinous band of disintegrating host cells" (Anderson and Rankin, 1914), "wound periderm” (Bramble, 1936), "macerated tissue" (McCarroll and Thor, 1978), and, "densely stainable hyphae" (Hebard, Griffin and Elkins, 1984). In trial studies, the gelatinous zone could not be located in dormant stems, in cankers that were not actively expanding, or in cankers that had been treated with hypovirulent inoculum in the previous fall. A combination of culture and staining work was required to resolve the discrepancies in descriptions of the gelatinous zone, but that work remains to be completed.

Barring the production of chestnut blight-resistant American chestnut trees, elucidating how hypovirus agents are affecting reproduction and other population dynamics of $C$. parasitica is likely the next step in successful use of hypoviruses in biological control efforts. Effective disease management using hypoviruses will require a 
better understanding of how they interact with and convert their host fungal population and how this translates into positive tree responses. If hypoviruses are unable to transmit, colonize, and replicate in established, virulent mycelium their use as biological control agents will be severely limited until the reservoir of virulent fungi is replaced with reproductively stable hypovirulent isolates. What potential for vertical transmission of the control agent exists when the hypovirulent fungus faces negative selection pressures ranging from reduced growth, pathogenicity, and sporulation while the virulent phenotype remains intact? Filamentous fungi have been described eloquently to form extensive "communication networks" from hyphal anastomoses (Rayner, 1991) but it is unknown whether or not fungi have active or passive mechanisms to shut the system down when it stands to benefit them, such as limited infections by a mycovirus. Lack of hypovirus colonization of reproductive structures even when underlying mycelium is hypovirulent (Shain and Miller, 1992), lack of accumulation of hypovirus in older hyphae such as with similar hypoviruses (Hansen, et al., 1985), and results from cultural work as part of this study all seem to indicate establishment of virulent fungus is the endpoint and only growth subsequent to hypovirus introductions is subject to the hypovirulent phenomenon. Further research into how hypoviruses are packaged into vesicles and move within host thallus should help elucidate why hypoviruses are easier to obtain for canker and colony margins rather than interior mycelium. 


\section{CONCLUSIONS}

Hypovirulent inoculum challenges to colony leading edges resulted in high "conversion" to the hypovirulent phenotype following challenge, not to mycelium established prior to challenge.

Even twelve weeks after challenge, established, virulent mycelium fails to yield hypovirulent isolates.

When colonies are challenged behind the colony leading edge, hypovirus can be transmitted through the existing mycelium, which did not always acquire the hypovirulent phenotype, even though newly extending mycelium did.

Up to one year following successful transmission of hypovirus, cankers may yield vegetatively compatible virulent or hypovirulent isolates from any portion of the canker regardless of whether the sampling point is from older or younger portions of cankers.

The relative recovery of hypovirulent isolates from cankers appears to decrease as you sample progressing in towards cankers centers and outwards as cankers expand following hypovirulent inoculum challenges.

Single bark plugs sectioned with a scalpel into smaller pieces can yield both virulent and hypovirulent isolates. 


\section{LITERATURE CITED}

Anagnostakis, S. L. 1987. Chestnut blight: The classical problem of an introduced pathogen. Mycologia. 79(1):23-27.

Anagnostakis, S. L. 1993. Chestnuts and the introduction of chestnut blight. Northern Nut Growers. Pages 1-11.

Anagnostakis, S. L. and Waggoner, P. E.1981. Hypovirulence, vegetative incompatibility, and the growth of cankers of chestnut blight. Phytopathology. 71:1198-1202.

Anderson, P. J. 1914. The morphology and life history of the chestnut blight fungus. Pennsylvania Chestnut Tree Blight Commission, Bulletin 7:1-44.

Anderson, P. J. and Babcock, D. C. 1913. Field studies on the dissemination and growth of the chestnut blight fungus. Pennsylvania Chestnut Tree Blight Commission, Bulletin 3:1-45.

Anderson, P. J. and Rankin, W. H. 1914. Endothia canker of chestnut. Bulletin 347, Cornell University Agricultural Experiment Station. Ithaca, NY. pp. 529-618.

Biraghi, A. 1946. Il cancro del castagno causato da Endothia parasitica. Ital. Agric. 7:1-9.

Bramble, W. C. 1936. Reaction of chestnut bark to invasion by Endothia parasitica. American Journal of Botany. 23:89-94.

Day, P.R., Dodds, J.A., Elliston, J.E., Jaynes, R.A., and Anagnostakis, S.L. 1977. Double-stranded RNA in Endothia parasitica. Phytopathology 67:1393-1396.

Elliston, J. E. 1982. Hypovirulence in Endothia parasitica and suggested procedures for its detection and analysis. Pages 1-13 Proceedings. USDA Forest Service American Chestnut Cooperators' Meeting. H.C. Smith and W.L. MacDonald, eds. West Virginia University Books, Morgantown, WV.

Fulbright, D. W., Weidlich, W. H., Haufler, K. Z., Thomas, C. S., and Paul, C. P. 1983. Chestnut blight and recovering American chestnut trees in Michigan. Canadian Journal of Botany. 61:3164-3171.

Hebard, F. V., Griffin, G. J., and Elkins, J. R. 1984. Developmental histopathology of cankers incited by hypovirulent and virulent isolates of Endothia parasitica on susceptible and resistant chestnut stems. Phytopathology 74:140-149. 
Heiniger, U and Rigling, D. 1994. Biological control of chestnut blight in Europe. Annual Review of Phytopathology. 32:581-599.

Keefer, W. E. 1914. Pathological histology of the Endothia canker of chestnut. Phytopathology. 4:191-200.

Kowalik, T.F. 1990. Clamp-R protocol for RT-PCR. Virology. 177:820-823.

Kuhlman, E. G. 1978. The devastation of American chestnut by blight. Pages 1-3 in: Proceedings of the American Chestnut Symposium. W. L. MacDonald, F. C. Cech, J. Luchok, and H. C. Smith, eds. West Virginia University Books, Morgantown, WV.

Lecellier, G. and Silar, P. 1994. Rapid methods for nucleic acids extraction from Petri dish-grown mycelia. Current Genetics. 25:122-123.

MacDonald, W. L. and Fulbright, D. W. 1991. Biological control of chestnut blight: Use and limitations of transmissible hypovirulence. Plant Disease. 75:656-661.

Martin, R.M. and Val Alfen, N.K.. 1991. The movement of viral-like RNA between colonies of Cryphonectria parasitica. Molecular Plant-Microbe Interactions 4:507-511.

McCarroll, D.R. and Thor, E. 1978. "Death of a Chestnut: The host pathogen interaction." p. 25-26 in Proceedings of the American Chestnut Symposium. W.L. MacDonald, et al.(eds.). West Virginia University Press, Morgantown. 122 p.

McManus, P. S., Ewers, F. W., and Fulbright, D. W. 1989. Characterization of the chestnut blight canker and the localization and isolation of the pathogen Cryphonectria parasitica. Canadian Journal of Botany. 67:3600-3607.

Murrill, W. A. 1906. A serious chestnut disease. Journal of the New York Botanical Garden. 7:143-153.

Newhouse, J.R. and MacDonald, W.L. 1991. The ultrastructure of hyphal anastomoses between vegetatively compatible and incompatible virulent and hypovirulent strains of Cryphonectria parasitica. Can. J. Bot. 69:602-614.

Papp, T., Nyilasi, I., Fekete, C., Ferenczy, L., and Vagvolgyi, C. 2001. Presence of double-stranded RNA and virus-like particles in Rhizopus isolates. Canadian Journal of Microbiology. 47:443-447.

Rankin, W. H. 1914. Field studies on the Endothia canker of chestnut in New York State. Phytopathology. 4:233-261.

Rayner, A.D. 1991. The challenge of the indivualistic mycelium. Mycologia. 83:48-71. 
Roane, M. K., Griffin, G. J., and Elkins, J. R. 1986. Chestnut blight, other Endothia diseases and the Genus Endothia. St. Paul, MN: APS Press. 53 pages.

Shain, L. and Miller, J. B. 1992. Movement of cytoplasmic hypovirulence agents in chestnut blight cankers. Canadian Journal of Botany. 70:557-561.

Shigo, A.L. 1967. Successions of Organisms in Discoloration and Decay of Wood. International Review of Forestry Research Volume 2. pp. 237-299.

Van Alfen, N.K., Jaynes, R.A., Anagnostakis, S.L., and Day, P.R. 1975. "Chestnut blight: Biological control by transmissible hypovirulence in Endothia parasitica." Science. 189:890-891.

Varga, J., Rinyu, E., Kevei, E., Toth, B., and Kozakiewicz, Z. 1998. Double-stranded RNA mycoviruses in species of Aspergillus sections Cirumdati and Fumigati. Candian Journal of Microbiology. 44:569-574.

Weidlich, W. H. 1978. "A preliminary report on a method of biological control of the chestnut blight not involving the use of a hypovirulent strain of Endothia parasitica." p. 25-26 in Proceedings of the American Chestnut Symposium. W.L. MacDonald et al.(eds). West Virginia University Press, Morgantown. 122 p. 


\section{APPENDIX A \\ Method for Verification of Phenotypic Data from Culturing Work}

Data for all three experiments in this research project were obtained by culturing Cryphonectria to score colonies as virulent, hypovirulent or contaminated based on cultural morphology and pigmentation. These determinations were facilitated by comparing them to standard cultures. Therefore, one weakness of the data was thought to be a lack of genotypic information about the isolates. To address this problem, additional samples were cultured and scored as was typical of this study but also were further analyzed using RT-PCR amplification of DNA extracted from mycelium and agar samples by Lynn Geletka with Dr. Donald Nuss at the University of Maryland. This method was felt to be an appropriate test of the phenotypic data as a single strand of hypovirus should be amplified if it were physically present but invisible due to its presence below a threshold level that may be required to elicit the hypovirulent phenotype.

Samples were obtained from standard cultures, a two-week old colony of MC2 that was challenged with hypovirulent inoculum at the colony leading edge one week earlier (Figure 18), and a three-week old colony of MC2 that was challenged one week prior (Figure 19). Colonies were established, challenged and sampled as described in Experiment I. Two mycelium and agar plugs were taken at each of 12 sample points to yield one sample for culturing to determine phenotype (e.g. orange-pigmented hypovirulent) and the other for the genotypic work. Samples of control isolates were obtained from the leading edge of one-week old colonies that appeared contaminant-free.

DNA for loading onto gels was obtained using the freeze-thaw method for extracting double-stranded nucleic acid from Petri dish-grown mycelium (Lecellier and Silar, 1994) as adapted for Cryphonectria (Geletka, 2000. pers. comm..). Amplification of DNA was achieved via a modified protocol Clamp-R RT-PCR (Kowalik, 1990). RTPCR gene products were visualized and photographed on May 5, 2000 following electrophorisis on $0.8 \%$ Seakem LE (BioWhittaker, Rockland, MD) agarose gel. 
Lanes 1 and 16 (Figure 18) and 1 and 17 (Figure 19) show the RT-PCR products from $1 \mathrm{~Kb}$ Plus DNA Ladders. The RT-PCR products from orange-pigmented virulent isolate $\mathrm{MC} 2$, brown pigmented hypovirulent isolate 80-2C [CHV1 80-2], and orange pigmented hypovirulent isolate EP 713 [CHV1 Euro 7] standards are shown in lanes 2, 3 and 16, respectively (Figure 18). Standards in Gel B are positive control EP 713 (Lane 1), virulent isolate MC2 (Lane 15) and hypovirulent isolate 80-2C [CHV1 80-2] (Lane 16). Lanes 4 through 15 (Figure 18) and lanes 3-14 (Figure 19) depicts the gel products from 12 mycelium and agar samples. In Gel A lanes 4, 5, 6 and 7 and lanes 3-14 in Gel $B$ depict the genotype of samples taken from mycelium established prior to hypovirus challenge. Lanes 8-15 in Gel A show the RT-PCR products from sampling of mycelium established subsequent to hypovirulent inoculum challenge.

In all cases, isolates scored as hypovirulent yielded a strong band at approximately $370 \mathrm{bp}$ while none of the samples scored as virulent contained this band (Figures 18 and 19). A faint to very faint band at $2100 \mathrm{bp}$ was seen in virulent standards and isolates but was thought to be an artifact from the large amount of fungal DNA binding weakly to the primers (Geletka, 2000. pers. comm.). In all samples tested the virulent or hypovirulent phenotype matched the genotype. 


\section{Gel A}

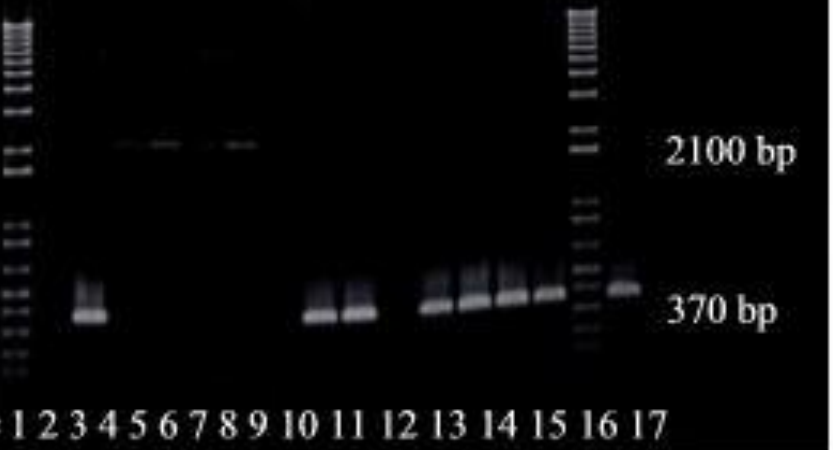

Figure 18: Digital scan of the RT-PCR products of $1 \mathrm{~Kb}$ plus DNA ladder (Lanes 1 and 16), standard virulent and hypovirulent isolates (Lanes 2, 3 and 17) and phenotypically virulent and hypovirulent isolates (Lanes 4-15) on 0.8\% agarose gel.

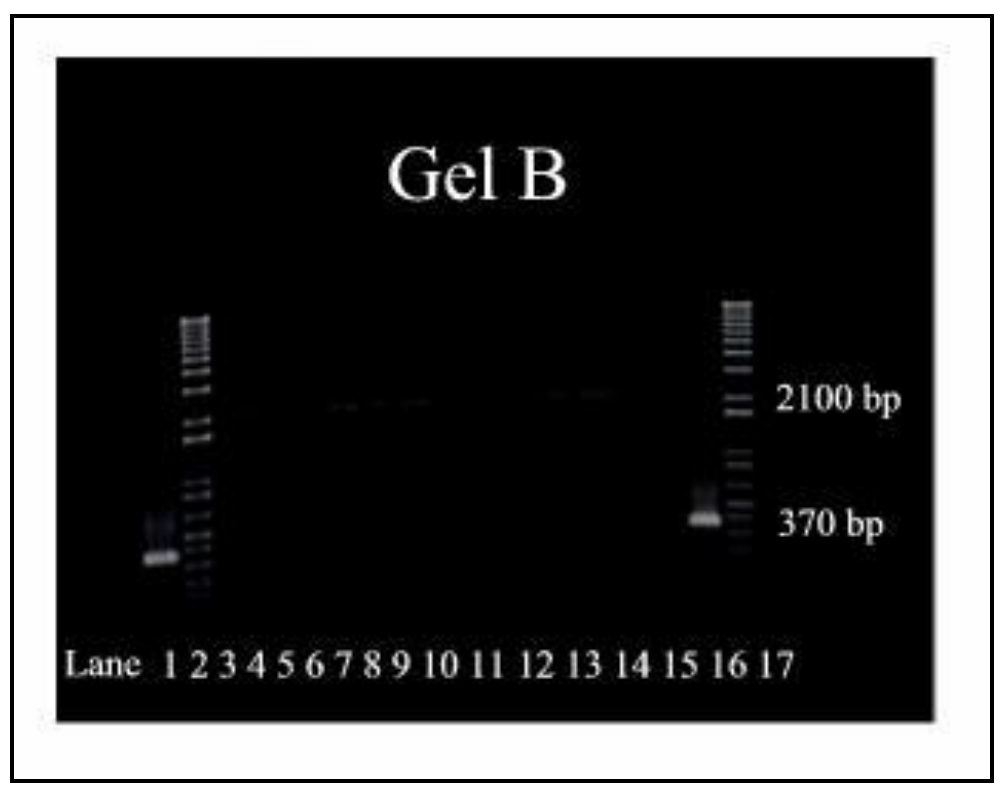

Figure 19. Digital scan of the RT-PCR products of $1 \mathrm{~Kb}$ plus DNA ladder (Lanes 2 and 17), standard virulent and hypovirulent isolates (Lanes 1,15 and 16) and phenotypically virulent and hypovirulent isolates (Lanes 3-14) on $0.8 \%$ agarose gel. 


\section{APPENDIX B: GROWTH MEDIA FORMULATIONS}

\section{GLUCOSE YEAST EXTRACT (GYE)}

\begin{tabular}{ll} 
Milli-Q® Water & $1.0 \mathrm{l}$ \\
Agar (Difco) & $20.0 \mathrm{~g}$ \\
Glucose & $10.0 \mathrm{~g}$ \\
Yeast Extract & $2.0 \mathrm{~g}$ \\
$\mathrm{KH}_{2} \mathrm{PO}_{4}$ & $1.0 \mathrm{~g}$ \\
$\mathrm{Mg}_{2} \mathrm{SO}_{4}$ & $0.5 \mathrm{~g}$ \\
Microelements* & $2.0 \mathrm{mg}$ \\
\multicolumn{1}{c}{ *Ferrous Sulfate } & $0.5 \mathrm{mg} / \mathrm{ml}$ \\
$\quad$ *Manganous Sulfate & $0.44 \mathrm{mg} / \mathrm{ml}$ \\
$\quad$ *Zinc Sulfate & $0.15 \mathrm{mg} / \mathrm{ml}$ \\
Biotin (0.005 mg/ml) & $1.0 \mathrm{ml}$ \\
Thiamine (0.001 mg/ml) & $1.0 \mathrm{ml}$ \\
Tetracycline Hydrochloride & $50.0 \mathrm{mg}$ \\
Streptomycin Sulfate & $6.0 \mathrm{mg}$
\end{tabular}

\section{POTATO DEXTROSE AGAR (PDA)}

$\begin{array}{ll}\text { Milli-Q® Water } & 1.0 \mathrm{l} \\ \text { PDA (Difco) } & 39.0 \mathrm{~g} \\ \text { Biotin }(0.005 \mathrm{mg} / \mathrm{ml}) & 1.0 \mathrm{ml} \\ \text { 1-L Methionine } & 0.1 \mathrm{~g} \\ \text { Tetracycline Hydrochloride } & 50.0 \mathrm{mg} \\ \text { Streptomycin Sulfate } & 6.0 \mathrm{mg}\end{array}$




\section{CURRICULUM VITAE}

William Edward Jones was born in 1973 in Doylestown, Pennsylvania to Gordon Mark and Helen Judith Jones. After New Hope-Solebury Jr./Sr. High School he attended West Virginia University and earned a Bachelor of Science degree in Forest Resources Management in 1996. In 1997, he began work on a Master of Science degree in Plant \& Soil Sciences with a focus in forest pathology at West Virginia University. He is currently employed as Plant Pathologist with State \& Private Forestry, Forest Health

Protection, USDA Forest Service located at Asheville, NC in the Southern Research Station working on hazard tree management, beech bark disease, dogwood anthracnose and other forest health problems. 